\title{
VARIABLE-COMPLEXITY RESPONSE SURFACE APPROXIMATIONS FOR WING STRUCTURAL WEIGHT IN HSCT DESIGN
}

\author{
By \\ Matthew Douglas Kaufman \\ A THESIS SUBMITTED TO THE FACULTY OF \\ VIRGINIA POLYTECHNIC INSTITUTE AND STATE UNIVERSITY \\ IN PARTIAL FULFILLMENT OF THE REQUIREMENTS FOR THE DEGREE OF \\ MASTER OF SCIENCE \\ IN \\ Aerospace Engineering
}

Bernard Grossman, Co-Chairman

Raphael T. Haftka, Co-Chairman

William H. Mason

Layne T. Watson

April 1996

Blacksburg, Virginia 


\title{
Abstract
}

\author{
VARIABLE-COMPLEXITY RESPONSE SURFACE APPROXIMATIONS \\ FOR WING STRUCTURAL WEIGHT IN HSCT DESIGN
}

\author{
by \\ Matthew Douglas Kaufman \\ Committee Chairs: Bernard Grossman and Raphael T. Haftka \\ Aerospace Engineering
}

(Abstract)

A procedure for generating and using a polynomial approximation to wing bending material weight of a High Speed Civil Transport (HSCT) is presented. Response surface methodology is used to fit a quadratic polynomial to data gathered from a series of structural optimizations. Several techniques are employed in order to minimize the number of required structural optimizations and to maintain accuracy. First, another weight function based on statistical data is used to identify a suitable model function for the response surface. In a similar manner, geometric and loading parameters that are likely to appear in the response surface model are also identified. Next, rudimentary analysis techniques are used to find regions of the design space where reasonable HSCT designs could occur. The use of intervening variables along with analysis of variance reduce the number of polynomial terms in the response surface model function. Structural optimization is then performed by the program GENESIS on a 28-node Intel Paragon. Finally, optimizations of the HSCT are completed both with and without the response surface. 


\section{Acknowledgements}

This work has been supported by NASA Grant NAG1-1562 with Dr. P. Newman as contract monitor and NASA Grant NAG1-1160 with Mr. P. Coen as contract monitor.

I would like to express my appreciation to those who encouraged me to pursue my graduate degree, Dr. Cornelis van Dam, Dr. Mohamed Hafez, and Dr. Allan McKillop. While working on my research, I received a significant amount of help from other graduate students. In particular, Anthony Giunta, Vladimir Balabanov and Peter MacMillin.

Finally, many thanks go to my family and friends who kept me smiling while I

finished school and even more gratitude to those who visited me during my stay in Virginia. 


\section{Contents}

$\begin{array}{ll}\text { Abstract } & \text { ii }\end{array}$

Acknowledgements

1 Introduction 1

2 Response Surface Methods $\quad 5$

3 HSCT Design Problem $\quad 8$

4 Aircraft Weight Function 13

5 Response Surface Approximation 16

5.1 The Model Function . . . . . . . . . . . . . . . . . 16

5.2 Identification of the Reasonable Design Space . . . . . . . . . 17

5.3 Selection of Intervening Variables . . . . . . . . . . . . . . . . 22

5.4 Regression Analysis and ANOVA . . . . . . . . . . . . . 24

6 Structural Optimization 26

6.1 Structural Optimization Model . . . . . . . . . . . . . 26

6.2 Noise in Structural Optimization _ . . . . . . . . . . . 28

6.3 Parallelized Structural Optimization _ . . . . . . . . . . 30

$\begin{array}{lll}7 & \text { RSM for Structural Optimization } & 32\end{array}$

8 Response Surface Implementation $\quad 40$ 
9 HSCT Design Optimization

10 Concluding Remarks $\quad 52$

A Least Squares Method and Analysis of Variance 59

B FLOPS Weight Function Details $\quad 61$

C Generation of Candidate Designs 63

D Response Surface Descriptions $\quad 65$

$\begin{array}{ll}\text { E Optimal HSCT Designs } & 72\end{array}$

$\begin{array}{lll}\text { F } & \text { RSG Program } & 77\end{array}$

F.1 Data Input . . . . . . . . . . . . . . . . . . 77

F.2 Data Pre-Processing . . . . . . . . . . . . . . . . . 79

F.3 Response Surface Generation . . . . . . . . . . . . . . . . 81

F.4 Data Output . . . . . . . . . . . . . . . 82

F.5 RSG Input Deck . . . . . . . . . . . . . . . . . . . . 83

$\begin{array}{ll}\text { G Vitae } & 88\end{array}$ 


\section{List of Tables}

3.1 HSCT baseline design variables. . . . . . . . . . . . . . . . 11

3.2 Optimization constraints. . . . . . . . . . . . . . . . . . 12

4.1 Basic parameters used to calculate wing weight in FLOPS. . . . . . 15

5.1 Criteria for reasonable designs. . . . . . . . . . . . . . . . . 19

5.2 Reduced design space response surface errors. . . . . . . . . . . 21

5.3 Accuracy of various RS models. . . . . . . . . . . . . . . 23

7.1 Apparent accuracy of various RS models. . . . . . . . . . . 38

9.1 Numerical comparison of $W_{g}$ and $W_{b}$ for each optimal HSCT design. . 45

9.2 Apparent error of $W_{b}$ values at each optimal HSCT design. . . . . . . 47

9.3 Optimal HSCT designs using response surface RS1. . . . . . . . . . 49

9.4 Optimal HSCT designs using response surface RS2 . . . . . . . 50

9.5 Optimal HSCT designs using response surface RS3 . . . . . . . 51

C.1 Two-variable block pattern example. . . . . . . . . . . . . . . . 64

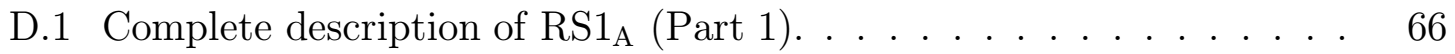

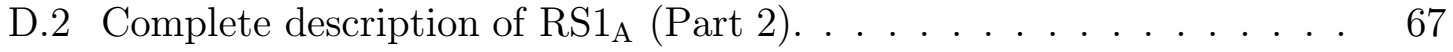

D.3 Complete description of $\mathrm{RS1}_{\mathrm{AB}}($ Part 1$) \ldots \ldots \ldots \ldots \ldots \ldots$

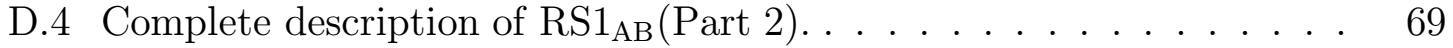

D.5 Complete description of $\mathrm{RS}_{\mathrm{A}} \ldots \ldots \ldots \ldots \ldots \ldots \ldots \ldots$

D.6 Complete description of $\mathrm{RS} 2_{\mathrm{AB}} \ldots \ldots \ldots \ldots \ldots \ldots \ldots$

D.7 Complete description of $\mathrm{RS}_{\mathrm{A}} \ldots \ldots \ldots \ldots \ldots \ldots \ldots \ldots \ldots$

D.8 Complete description of $\mathrm{RS} 3_{\mathrm{AB}} \ldots \ldots \ldots \ldots \ldots \ldots \ldots$

E.1 Design variables for optimal HSCT design using FLOPS. . . . . . . 73

E.2 Design variables for optimal HSCT design using RS1. . . . . . . . . 74 
E.3 Design variables for optimal HSCT design using RS2 . . . . . . . 75

E.4 Design variables for optimal HSCT design using RS3. . . . . . . 76 


\section{List of Figures}

3.1 HSCT wing planform and airfoil parameters. . . . . . . . 9

5.1 Sample planforms after the exclusion of infeasible geometries. . . . . 19

5.2 Sample planforms after the exclusion of unreasonable geometries. . . 20

5.3 Sample planforms after the exclusion of designs with insufficient range. 20

5.4 RS error versus terms remaining in design variable model function. . 24

5.5 RS error versus terms remaining in intervening variable model function. 25

6.1 Typical structural optimization model. . . . . . . . . . . . . . . 27

6.2 Comparison of FLOPS with structural optimization results. . . . . 28

6.3 Comparison of extremum designs used for noise analysis. . . . . . . 29

6.4 Ideal versus actual speedup for parallel execution of the GENESIS. . 31

7.1 Apparent accuracy of full RS in design and intervening variables. . . 34

7.2 RS apparent error versus terms in design variable model function. . . 35

7.3 RS apparent error versus terms in intervening variable model function. 35

7.4 Reduced apparent error using ANOVA and design variable RS. . . . 36

7.5 Reduced apparent error using ANOVA and intervening variable RS. . 36

7.6 Apparent accuracy of reduced RS in design and intervening variables. 37

9.1 Comparison of $W_{g}$ and $W_{b}$ for each optimal HSCT design. $\ldots \ldots .45$

9.2 Optimal HSCT planforms using RS1. . . . . . . . . . . . . 49

9.3 Optimal HSCT planforms using RS2 . . . . . . . . . . . 50

9.4 Optimal HSCT planforms using RS3. . . . . . . . . . . . . 51

F.1 RSG standard model function file example. . . . . . . . . . . . . 79

F.2 RSG data file example. . . . . . . . . . . . . . . . . . 80 


\section{Chapter 1}

\section{Introduction}

The use of multidisciplinary optimization techniques in aerospace vehicle design is often limited because of the significant computational expense incurred in the analysis of the vehicle and its many systems. In response to this difficulty, a variable-complexity modeling approach involving the use of refined and computationally expensive models together with simple and computationally inexpensive models has been developed. This variable-complexity technique has been previously applied to the combined aerodynamic-structural optimization of subsonic transport aircraft wings [1] and the aerodynamic-structural optimization of the High Speed Civil Transport (HSCT) $[2,3]$.

In this study, the integration of a response surface approximation to wing bending material weight into the HSCT configuration design process is considered. Response surface methodology (RSM) was first developed by statisticians $[4,5,6]$ and has more recently been applied to the design aerospace vehicles. A sizing study for a stopped rotor/wing aircraft configuration utilized response surfaces to model the coupled rotor/engine sizing, where individual components could not be studied in isolation [7]. In other work, response surfaces were used to find an optimal aerodynamic configuration for a single-stage-to-orbit re-entry vehicle [8]. For HSCT design, response surfaces have been used to construct smooth approximations to aerodynamic drag components whose calculations were susceptible to numerical noise $[9,10]$.

Previous work involving the HSCT wing bending material weight relied on one of two independent weight functions to estimate bending material weight as a function 
of the design variables [11]. Here, a weight function refers to a series of algebraic equations, formulated using experience-based statistical information. Since the HSCT is a new class of aircraft, weight functions do not account for all features of the design. In a comparison with finite-element-based structural optimization the weight function from the Flight Optimization System (FLOPS) [12] and from a Grumman Aerospace Corporation contractor report [13] were both found suitable for predicting general trends in structural weight, but unable to accurately model all effects of changing aircraft geometry. When compared to structural optimization, the FLOPS weight function was found to be more accurate than the Grumman weight function [14]. For this reason, the Grumman weight function is not used in this work; however, the FLOPS weight function will be used extensively and will now be reffered to as the weight function.

Following the variable-complexity modeling approach, information from structural optimization was incorporated into the weight function. This was accomplished by generating a scale factor which multiplied the bending material part of the weight function to match structural optimization results [15]. This procedure improved the accuracy of the weight function, but derivatives of the scale factor were not available to the configuration design optimizer, making optimal solutions difficult to find. In addition, the scale factor was only updated periodically during the design due to the cost associated with structural optimization.

In view of the weight function deficiencies, a full integration of the structural and configuration optimization was considered. However, this approach was difficult for several reasons. First, results from the structural optimization did not produce smooth functions with respect to the configuration shape parameters. Therefore, a derivative-based optimization would have been difficult to perform. Second, the configuration design process required structural weight information at a large number of design points. This, coupled with the expense of structural optimization, made an integrated optimization infeasible for HSCT design, where multiple optimizations must be performed.

Coupling unrelated optimization processes also introduced code integration problems. Different optimization software packages utilized different design variables, 
input parameters, and output formats. Additional software, commonly termed wrappers, could have been developed to automate code interaction; however, this is rarely a straightforward process. Moreover, wrappers produce inefficiencies on modern high performance computers. Examples of research in this area are described in $[16,17]$.

In this thesis, a response surface approximation to the wing bending material weight is used to address the concerns of function smoothness, cost, and code integration, while also improving the accuracy of the statistical weight function. Instead of performing structural optimization during the configuration design process, a large number of aircraft geometries are evaluated beforehand. These results are then used to create a response surface to the wing bending material weight. Since the geometries are based on the configuration design variables, code integration problems are eliminated.

While one desires accurate results throughout the entire design space, it is impractical to perform structural optimization for every conceivable HSCT configuration. For this reason, techniques are developed to limit the design space domain and to balance the response surface accuracy with development cost. These methods are not specific to the wing bending material weight and can be applied to many response surface applications.

Three methods specifically improve the accuracy of the response surface. First, a good model function is found by inspecting various statistical weight functions. Second, inexpensive approximate analysis methods are used to find regions of the design space where reasonable HSCT configurations are likely to appear. Development of the response surface is then limited to this portion of design space. Third, analysis of variance is used to remove unnecessary terms from the model function [6]. The absence of these terms reduces the incidence of large errors in the final response surface.

The cost of generating the response surface is addressed in several ways. Once again, a previously developed statistical weight function is used to identify a small set of geometric and loading parameters which characterize the wing bending material weight objective function. This reduced set of variables is then used in place of the HSCT design variables to reduce the complexity of the model function. Finally, using 
the $D$-optimality criterion, a small number of reasonable designs, which produce a response surface that accurately reflect the entire reasonable design space, are chosen [18]. Only at these designs are structural optimizations performed.

The large number of structural optimizations required make this problem especially suitable for coarse grain parallelization. A finite-element-based structural optimization code, GENESIS, is used to optimize the numerous design configurations [19]. GENESIS has been modified for the Intel Paragon and reasonable speedups are achieved.

By its nature, the response surface is a simple algebraic expression that provides smooth derivative information. Although initially expensive to establish, the surface uses minimal resources once implemented. This makes it ideal for the HSCT design problem. To demonstrate the response surfaces' suitability to design, optimizations of the HSCT configurations are performed. Results are presented with and without the implementation of the response surface. 


\section{Chapter 2}

\section{Response Surface Methods}

Response surface methodology (RSM) is a statistical technique in which smooth functions, typically polynomials, are used to model an objective function. For example, a quadratic response surface model for $p$ variables has the form:

$$
y=c_{0}+\sum_{1 \leq i \leq p} c_{i} x_{i}+\sum_{1 \leq i \leq j \leq p} c_{i j} x_{i} x_{j},
$$

where the $x_{i}$ are the variables, the $c_{i}$ are the polynomial coefficients, and $y$ is the measured response. For $p$ variables, $(2.1)$ has $n=(p+1)(p+2) / 2$ terms. In such a model the polynomial coefficients may be estimated using the method of least squares (Appendix A). Throughout this work, the RSG program is used to generate response surfaces (Appendix F).

Most RSM applications are based on quadratic polynomials. While these polynomials are relatively easy to utilize, some issues must be considered before their implementation. For $p$ variables, the number of terms in the model function, $n$, grows at a rate $\mathcal{O}\left(p^{2}\right)$. Creating a response surface for the $n$-term polynomial requires a number of points, $m$, which is larger than, but of the same order of magnitude as $n$. This seems reasonable; however, to maintain good accuracy, one needs to minimize estimations beyond the domain where analyses were performed. That is, one would like the

points where the response surface is used to reside within the convex hull of the data points used to construct the surface. To ensure this condition for a $p$-dimensional box requires at least $2^{p}$ points (at the vertices of the box). For the twenty-eight design 
variables which describe the HSCT, this corresponds to more than 200 million points, which is certainly impossible with present computational capabilities. This problem is often referred to as the curse of dimensionality. Quadratic polynomials also of course cannot model higher order variations.

Estimations outside the design space where the response surface was created may produce accurate results; however, it is well known that if the model is wrong, estimates only slightly outside the known data can be grossly in error. Several measures can be taken to address model error. Although seldom an option, the number of data points used to create the response surface can be increased so that the convex hull defined by the known data points encloses a larger portion of the design space. Another option is to reduce the volume of the design space modeled by RSM. This will lower the distance between points exterior to the convex hull and the boundary of the convex hull. Finally, the complexity of the model function can be reduced by eliminating unnecessary terms. The statistical techniques, regression analysis and analysis of variance (ANOVA) enable the less significant terms in the polynomial approximation to be identified. ANOVA is described in Appendix A.

Computational resources limit the number of points from an ideal $2^{p}$ to the same order of magnitude as $n$. For a six-term polynomial in two dimensions, results from [20] indicate that $1.5 n$ function analyses are sufficient to produce a response surface where the error stabilize and the global trends of the underlying function are accurately approximated.

RSM typically employs a structured method such as central composite design (CCD) for selecting analysis points in the design space [21]. However, CCD is only effective for a low dimensional regularly shaped design space, which is unlikely to appear in this study. For an irregular design space in many dimensions, there is no simple way of creating a finite number of points that span the entire region. Hence, a very large number of points must be produced knowing that many will fall outside of the feasible design space. The infeasible points can then either be perturbed until they fall within the feasible region, or removed. This process will lead to a large number of points inside the feasible region, but whose geometric distribution is irregular. From these points, a small number must be chosen to construct the response surface. In a 
previous study [20], it was found that the $D$-optimal criterion [18] provided a rational means for choosing any number of these points.

Once the response surface is generated, its predictive capabilities must be evaluated. This is accomplished by finding the response surface prediction at a series of data points with known responses. Measuring the difference between the known and the predicted response yields the following information: average error, root mean square (RMS) error, and maximum error. Points used to evaluate the response surface should be different from the points used to create the response surface. If not, the error results may be misleading. 


\section{Chapter 3}

\section{HSCT Design Problem}

Successful aircraft configuration optimization requires a simple yet meaningful mathematical characterization of the geometry. The model used here completely defines the HSCT design problem using twenty-eight design variables [22, 23]. Twenty-five of the design variables describe the geometry of the aircraft and can be divided into five categories: wing planform, airfoil shape, tail areas, nacelle placement, and fuselage shape. The wing planform is described using the root and tip chord lengths, the wing span, and by blending linear line segments at the leading and trailing-edges. The airfoil sections have round leading edges and are defined using an analytic description that incorporates four variables. The horizontal and vertical tail areas are described by two variables. The nacelles move axially with the trailing-edge of the wing, and two variables define their spanwise locations. The axisymmetric fuselage requires eight variables to specify both the axial positions and radii of the four fuselage restraint locations. Figure 3.1 and Table 3.1 define the HSCT geometry and design variables.

In this study, a baseline HSCT is used to provide a point near the interior of the feasible design space. The baseline geometry is from an HSCT configuration previously thought to be optimal by Dudley et al, 1995 (Table 3.1). Because of modifications and improvements to the analysis methods, this geometry no longer satisfies all of the performance constraints; however, more recent optimal designs exhibit similar characteristics [24]. 


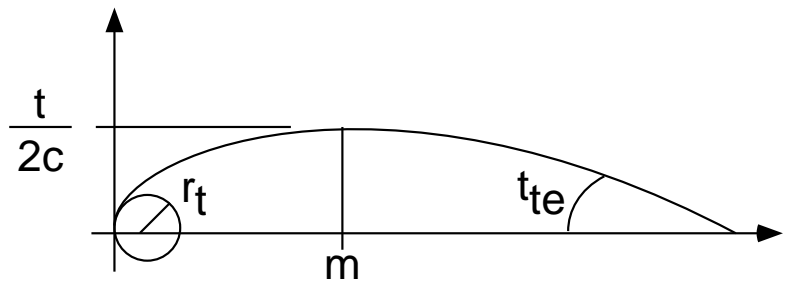

Airfoil Thickness Distribution Parameters

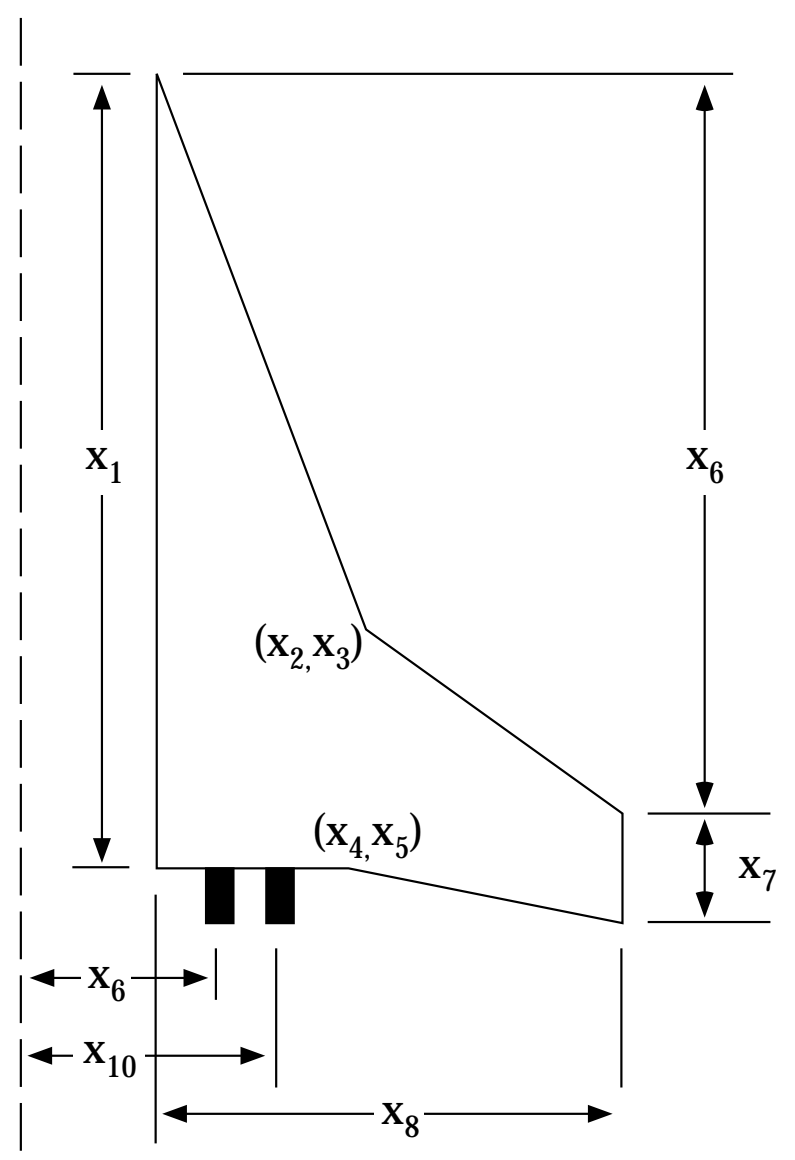

Wing Planform and Nacelle Placement Parameters

Figure 3.1: HSCT wing planform and airfoil parameters. 
The design problem is to minimize the takeoff gross weight of an HSCT configuration with a range of 5,500 nautical miles and a cruise speed of Mach 2.4 while transporting 251 passengers. For this mission, in addition to the geometric parameters, four variables define the idealized cruise mission (Table 3.1): fuel weight, initial cruise altitude, and the constant climb rate used in the range calculation.

A complete design optimization is composed of a sequence of optimization cycles. Detailed analysis methods are employed at the beginning of each cycle, while simple analyses, scaled to match the initial detailed results, are performed in subsequent calculations during each cycle $[2,3]$. A typical HSCT design requires approximately twenty-five cycles until an optimal configuration is identified. The optimizer NEWSUMT-A [25], which employs an extended interior penalty function method, is used for this work. NEWSUMT-A utilizes constraints based on both the simple and detailed analyses along with constraints that limit the movement of the design variables.

Sixty-eight constraints, including geometry, performance, and aerodynamic constraints, prevent the optimizer from creating physically impossible designs [23]. The aerodynamic and performance constraints can only be assessed after a complete analysis of the HSCT design; however, the geometric constraints can be evaluate using algebraic relations based on the twenty-eight design variables. For this reason, they offer a very efficient, albeit rudimentary, means of identifying unreasonable HSCT designs. Reasonable designs are defined to be those that exhibit similar characteristics to feasible designs even though some aerodynamic and performance constraints might be violated. For the most part, however, the geometric constraints only prevent nonsensical configurations such as those where the engine nacelles are not located on the wing or the airfoil chord lengths become negative. All sixty-eight constraints are listed in Table 3.2 . 
Table 3.1: HSCT baseline design variables.

\begin{tabular}{|r|c|r|l|}
\hline$\#$ & Value & Multiplier & \multicolumn{1}{|c|}{ Description } \\
\hline \hline 1 & 1.814780 & 100 & Wing root chord $(\mathrm{ft})$ \\
\hline 2 & 1.559070 & 100 & LE Break, $x(\mathrm{ft})$ \\
\hline 3 & 4.923480 & 10 & LE Break, $y(\mathrm{ft})$ \\
\hline 4 & 1.816030 & 100 & TE Break, $x(\mathrm{ft})$ \\
\hline 5 & 6.424340 & 10 & TE Break, $y(\mathrm{ft})$ \\
\hline 6 & 1.695730 & 100 & LE of wing tip, $x(\mathrm{ft})$ \\
\hline 7 & 0.700360 & 10 & Tip chord $(\mathrm{ft})$ \\
\hline 8 & 7.590870 & 10 & Wing semi-span $(\mathrm{ft})$ \\
\hline 9 & 4.019410 & 0.10 & Chordwise location of max. $t / c$ \\
\hline 10 & 3.692140 & 1 & Airfoil LE radius parameter, $r_{t}$ \\
\hline 11 & 2.578860 & 0.01 & Airfoil $t / c$ at root \\
\hline 12 & 2.159350 & 0.01 & Airfoil $t / c$ at LE break \\
\hline 13 & 1.803900 & 0.01 & Airfoil $t / c$ at tip \\
\hline 14 & 0.021960 & 100 & Fuselage restraint $1, x(\mathrm{ft})$ \\
\hline 15 & 0.106100 & 10 & Fuselage restraint $1, r(\mathrm{ft})$ \\
\hline 16 & 0.122000 & 100 & Fuselage restraint $2, x(\mathrm{ft})$ \\
\hline 17 & 0.349610 & 10 & Fuselage restraint $2, r(\mathrm{ft})$ \\
\hline 18 & 1.324630 & 100 & Fuselage restraint $3, x(\mathrm{ft})$ \\
\hline 19 & 0.534100 & 10 & Fuselage restraint $3, r(\mathrm{ft})$ \\
\hline 20 & 2.486680 & 100 & Fuselage restraint $4, x(\mathrm{ft})$ \\
\hline 21 & 0.466610 & 10 & Fuselage restraint $4, r(\mathrm{ft})$ \\
\hline 22 & 2.623010 & 10 & Nacelle $1, x_{9}(\mathrm{ft})$ \\
\hline 23 & 3.238760 & 10 & Nacelle $2, x_{10}(\mathrm{ft})$ \\
\hline 24 & 3.226170 & 100000 & Mission fuel $(\mathrm{lbs})$ \\
\hline 25 & 6.479430 & 10000 & Starting cruise altitude $(\mathrm{ft})$ \\
\hline 26 & 0.338980 & 100 & Cruise climb rate $(\mathrm{ft} / \mathrm{min})$ \\
\hline 27 & 6.978540 & 100 & Vertical tail area $\left(\mathrm{ft}{ }^{2}\right)$ \\
\hline 28 & 7.130590 & 100 & Horizontal tail area $\left(\mathrm{ft}{ }^{2}\right)$ \\
\hline & & & \\
\hline
\end{tabular}


Table 3.2: Optimization constraints.

\begin{tabular}{|c|c|}
\hline \# & Description \\
\hline 1 & Range $\geq 5,500$ \\
\hline 2 & Landing angle of attack $\leq 12^{\circ}$ \\
\hline 3 & Landing $\mathrm{CL} \leq 1.0$ \\
\hline $4-21$ & Landing section $\mathrm{Cl} \leq 2.0$ \\
\hline 22 & Fuel volume $\leq$ half of wing volume \\
\hline $23-40$ & Wing chord $\geq 7.0 \mathrm{ft}$ \\
\hline 41 & LE break, $y \leq$ wing semi-span \\
\hline 42 & TE break, $y \leq$ wing semi-span \\
\hline 43 & Root $t / c \geq 1.5 \%$ \\
\hline 44 & LE break $t / c \geq 1.5 \%$ \\
\hline 45 & TE break $t / c \geq 1.5 \%$ \\
\hline 46 & Fuselage: $x_{r e s t} \geq 5 f t$ \\
\hline 47 & Fuselage: $x_{\text {rest }_{1}}+10 \mathrm{ft} \leq x_{\text {rest }_{2}}$ \\
\hline 48 & Fuselage: $x_{r_{\text {est }}}+10 \mathrm{ft} \leq x_{\text {rest }_{3}}$ \\
\hline 49 & Fuselage: $x_{\text {rest }_{3}}+10 \mathrm{ft} \leq x_{\text {rest }_{4}}$ \\
\hline 50 & Fuselage: $x_{\text {rest }_{4}}+10 \mathrm{ft} \leq 300 \mathrm{ft}$ \\
\hline 51 & Nacelle $1, y \geq$ side-of-body \\
\hline 52 & Nacelle $1, y \leq$ nacelle $2, y$ \\
\hline 53 & Engine out stability criterion \\
\hline 54 & Minimum airfoil section spacing at wing tip \\
\hline $55-56$ & No engine scrape at landing angle-of-attack \\
\hline $57-58$ & No engine scrape at landing angle-of-attack, with $5^{\circ}$ roll \\
\hline 59 & No wing tip scrape at landing angle-of-attack, with $5^{\circ}$ rol \\
\hline 60 & $\begin{array}{l}\text { No wing trailing-edge break point scrape at landing, with } \\
5^{\circ} \text { roll }\end{array}$ \\
\hline 61 & Crosswind landing capability with aileron deflection only \\
\hline 62 & $\begin{array}{l}\text { Crosswind landing capability with aileron and rudder } \\
\text { deflection }\end{array}$ \\
\hline 63 & Tail deflection $\leq 22.5^{\circ}$ for landing \\
\hline 64 & $\begin{array}{l}\text { Takeoff rotation must occur prior to reaching } 90 \% \text { of } \\
\text { takeoff velocity }\end{array}$ \\
\hline 65 & No negative sweep of wing inboard trailing-edge \\
\hline 66 & $\begin{array}{l}\text { Root trailing-edge must not overlap root leading-edge of } \\
\text { horizontal tail }\end{array}$ \\
\hline $67-68$ & Required engine thrust $\leq$ available thrust \\
\hline
\end{tabular}




\section{Chapter 4}

\section{Aircraft Weight Function}

Gross takeoff weight is minimized during the HSCT configuration optimization process. Therefore, weight calculations are numerous and the final HSCT design is highly dependent on the accuracy of these results. To ease the computational expense of the design process, the statistical weight function in the weight module of Flight Optimization System (FLOPS) [12] has been implemented. FLOPS is used to determine takeoff gross weight and to find the effect of planform geometry changes on structural weight. However, since the HSCT is a new aircraft, the weight function does not account for all features of the design. Furthermore, by its nature, a weight function can only provide an approximate estimate of structural weight. The accuracy of the structural weight predictions from FLOPS is inadequate for HSCT design. This work focuses on the wing bending material weight since most of the load-dependent wing weight is due to bending.

The general wing weight function in FLOPS is based on an analytic expression to relate wing bending material weight to wing geometry, material properties, and loading. Other terms are added to account for shear material, control surfaces, etc. In addition, constants are included to correlate with a wide range of existing transports and to reflect features such as composite materials, strut braced wings, etc. The wing weight $W_{w}$ used within FLOPS is given as

$$
W_{w}=\frac{W_{g} K_{e} W_{b}+W_{s}+W_{n}}{1+W_{b},}
$$


where

$$
\begin{aligned}
W_{s} & =0.68\left(1-0.17 f_{c}\right)\left(S-S_{b}\right)^{0.34} W_{g}^{0.6}, \\
W_{n} & =0.35\left(1-0.3 f_{c}\right) S^{1.5} \\
W_{b} & =K f_{u l} b\left(1-0.4 f_{c}\right)\left(1-.01 f_{a}\right), \\
K & =8.8 B_{z}\left(1+(6.25 / b)^{0.5}\right) \times 10^{-6}, \\
K_{e} & =1.0-\left(B_{z e} / B_{e}\right)\left(W_{p o d} / W_{g}\right),
\end{aligned}
$$

and $W_{g}$ is the gross takeoff weight (lbs), $W_{b}$ is the wing bending material weight (lbs), $W_{s}$ is the wing shear material and flaps weight (lbs), $W_{n}$ is the wing control surfaces and non-structural weight (lbs), $W_{b}$ is the wing bending material weight (lbs), $W_{\text {pod }}$ is the engine pod weight (lbs), $b$ is the wing span (ft), $B_{z}$ is the bending material factor, $B_{z e}$ is the engine relief factor, $f_{u l}$ is the ultimate load factor, $f_{a}$ is the composite material factor, $f_{c}$ is the aeroelastic tailoring factor, $S$ is the wing area $\left(\mathrm{ft}^{2}\right)$, and $S_{b}$ is the wing box area $\left(\mathrm{ft}^{2}\right)$.

The system is closed except for the bending material factor $B_{z}$ and the engine relief factor $B_{z e}$. The parameter $B_{z}$ accounts for the distribution of load on the wing and is calculated by approximately determining the required material volume of the upper and lower skins in a simple wing box description of the wing. The parameter $B_{z e}$ accounts for the reduced amount of structural weight necessary due to the presence of the engines on the wing. Analytic expressions for $B_{z}$ and $B_{z e}$ are given in Appendix B. The FLOPS wing weight calculation is an iterative process since the wing weight and the gross takeoff weight are dependent on one another. Using a secant method, approximately seven iterations are required to converge.

A close inspection of the FLOPS weight function reveals that, for a design such as the HSCT, the wing weight is entirely based on a set of ten parameters, listed in Table 4.1. Each of these parameters can be found using the twenty-eight HSCT design variables. 
Table 4.1: Basic parameters used to calculate wing weight in FLOPS.

\begin{tabular}{|r|r|l|}
\hline$\#$ & Name & \multicolumn{1}{|c|}{ Description } \\
\hline \hline 1 & $S_{h t}$ & Horizontal tail surface area \\
\hline 2 & $S_{v t}$ & Vertical tail surface area \\
\hline 3 & $w f u s e$ & Maximum fuselage diameter \\
\hline 4 & $b$ & Wing span \\
\hline 5 & sweep & Average 1/4 chord sweep angle \\
\hline 7 & $B_{z}$ & Bending material factor \\
\hline 6 & $B_{z e}$ & Engine relief factor \\
\hline 8 & $S_{w}$ & Wing surface area \\
\hline 9 & $W_{f u e l}$ & Weight of fuel at takeoff \\
\hline 10 & $W_{t o}$ & FLOPS estimated gross weight \\
\hline
\end{tabular}




\section{Chapter 5}

\section{Response Surface Approximation}

An effective way to improve the estimate for the wing bending material weight given by FLOPS appears to be finite-element-based structural optimization. For this reason, an integration of the structural and configuration optimization was considered. However, problems with function smoothness, code integration, and limited computational resources prevented a combination of the two optimization processes. Instead, response surface methodologies are implemented to model the wing bending material weight calculated through structural optimization.

In the development of the response surface, a large number of data points spanning the design space are required; however, limits are imposed by the expense of performing structural optimization. For this reason, the bending material weight predicted by FLOPS is used to develop the response surface. Once this is complete, a sequence of structural optimizations is performed to generate the final form of the response surface.

\subsection{The Model Function}

The basic form of statistical weight functions such as those defined within FLOPS involve relationships such as

$$
y=C x_{1}^{c_{1}} x_{2}^{c_{2}} \cdots x_{p}^{c_{p}},
$$


where $y$ is an intermediate variable and the $x_{i}$ are the variables. This suggests a model equation of the form

$$
\ln (y)=c_{0}+\sum_{i=1}^{p} c_{i} \ln \left(x_{i}\right)+\sum_{1 \leq i \leq j \leq p} c_{i j} \ln \left(x_{i}\right) \ln \left(x_{j}\right) .
$$

Essentially, the logarithm of the response, $y$, is a quadratic function in the logarithms of the variables $x_{i}$. The $b$ coefficients are unknown values which must be estimated through the method of least squares (Appendix A). For comparison, model functions of both forms (2.1) and (5.2) are used to develop response surfaces to wing bending material weight.

\subsection{Identification of the Reasonable Design Space}

Three of the twenty-eight HSCT design variables - wing leading edge radius, cruise climb rate, and starting cruise altitude - have no effect on the FLOPS estimate for wing bending material weight, and so are ignored. The enormous design space associated with twenty-five variables, as well as the expense of performing structural optimization, forces one to limit the response surface to reasonable regions of the space. Again, a reasonable design refers to one whose characteristics are similar to a feasible design even though some of the aerodynamic and performance constraints might be violated.

The first step in identifying the reasonable design space is to construct a suitably large hypercube, defined by the twenty-five design variables (Table 3.1), that encompasses this entire region of space. Each of the variables, except the fuel weight, is allowed to assume values between $20 \%$ and $180 \%$ of its baseline value, given in Table 3.1. The fuel weight is only allowed to vary between $75 \%$ and $125 \%$ of its baseline value because of its strong influence on the design's range and therefore feasibility. Using the technique described in Appendix C, 19,651 configurations are found on the boundary of the domain. Of these designs, $83 \%$ violate one or more of the HSCT's geometric constraints (Table 3.2) and a large portion of the remaining designs appear to be unreasonable. 
Eliminating designs that are unreasonable cannot be accomplished without removing nearly every design in the pool of 19,651 candidate points. For this reason, each unreasonable design, $\mathbf{x}$ is moved so that it resides on the edge of the reasonable design space:

$$
\mathbf{x}^{\prime}=\alpha\left(\mathbf{x}-\mathbf{x}_{c}\right)+\mathbf{x}_{c}
$$

The parameter $\alpha, 0 \leq \alpha \leq 1$, is found using bisection.

Computing $\alpha$ in (5.3) requires a set of criteria to determine whether a design is reasonable or not. These criteria must be selected carefully to avoid a computationally expensive procedure and to ensure that no reasonable designs are inadvertently removed. In order to make use of complex constraints, a series of increasingly expensive evaluations are defined and applied in phases. Initially, the simple criteria are applied to the data and a large percentage of the candidate points are moved toward $x_{c}$. However, as the increasingly complex constraints are applied, fewer of the points have to be moved and the expense of the constraint evaluations does not become prohibitive.

Table 5.1 lists the criteria used to move the data towards the reasonable design space. They are listed in order of application, with the range constraint, which is the most expensive, coming last. During the first phase, the thirty-four geometric constraints listed in Table 3.2 are applied, resulting in the movement of 16,297 designs. Although these criteria do exclude geometrically impossible designs, they do not preclude unreasonable geometries. Originally, these constraints were intended to work in conjunction with the aerodynamic and performance constraints, which are not considered in this phase. Two unreasonable aircraft planforms that conform to the thirty-four constraints are depicted in Figure 5.1.

During the next phase of moving points towards the reasonable design space, the bending material weight $W_{b_{\mathrm{F}}}$ is computed by FLOPS. Based on past experience with the weight function, reasonable designs only occur when the FLOPS estimate for wing bending material weight $W_{b_{\mathrm{F}}}$ is between 20,000 lbs and 120,000 lbs, so designs falling outside that range are moved closer to the baseline design. This phase moves a total of 1,210 designs.

To further reduce the design space several new criteria are formulated to address 
Table 5.1: Criteria for reasonable designs.

\begin{tabular}{|r|l|}
\hline$\#$ & \multicolumn{1}{|c|}{ Description } \\
\hline \hline $1-34$ & HSCT geometric constraints (Table 3.2) \\
\hline $35-36$ & $20,000 \mathrm{lbs}<W_{b_{F}}<120,000 \mathrm{lbs}$ \\
\hline $37-58$ & Minimum fuselage radius \\
\hline 59 & Inboard $\Lambda_{l e}>$ Outboard $\Lambda_{l e}$ \\
\hline 60 & $\Lambda_{l e}>0$ \\
\hline $61-62$ & $5,000 \mathrm{ft}^{2}<S_{w}<15,000 \mathrm{ft}^{2}$ \\
\hline $63-64$ & $1.0<A R<3.2$ \\
\hline 65 & Inboard $\Lambda_{t e}<40^{\circ}$ \\
\hline $65-83$ & $c_{y_{i+1}} / c_{y_{i}}<1.0$ \\
\hline 84 & Approximate range $>5,000 \mathrm{n} . \mathrm{mi}$. \\
\hline
\end{tabular}
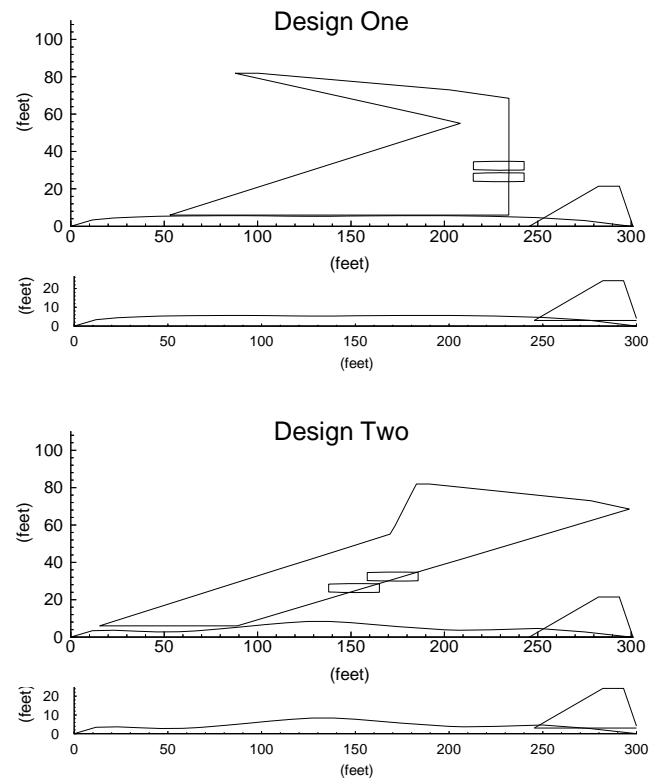

Figure 5.1: Sample planforms after the exclusion of infeasible geometries. 

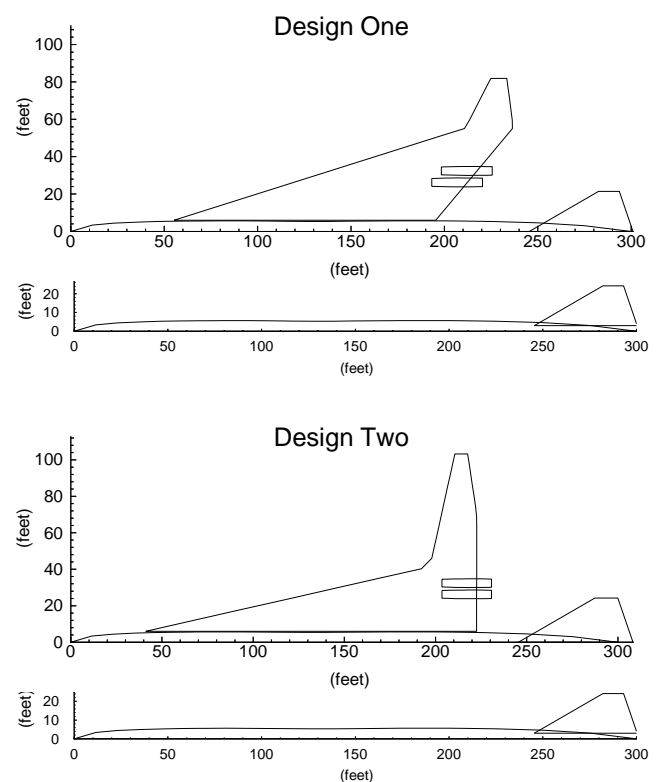

Figure 5.2: Sample planforms after the exclusion of unreasonable geometries.
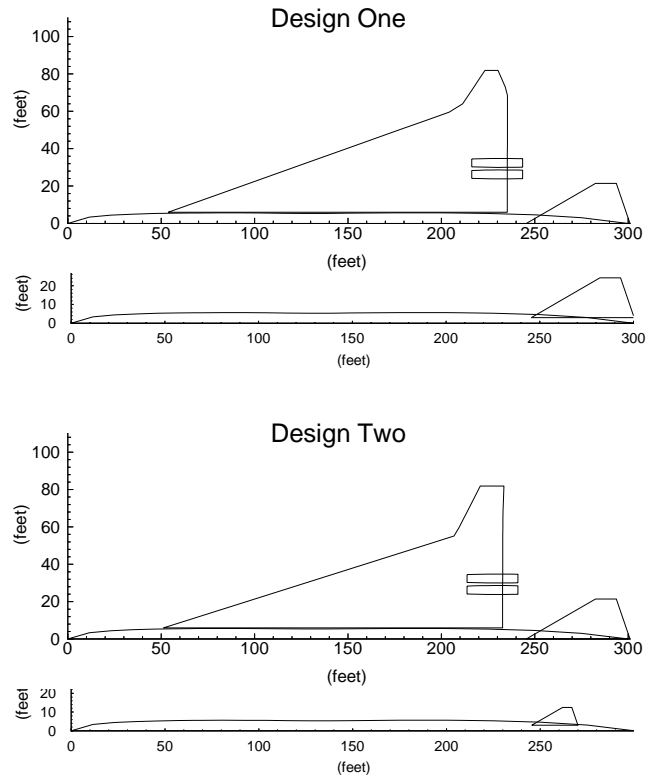

Figure 5.3: Sample planforms after the exclusion of designs with insufficient range. 
Table 5.2: Reduced design space response surface errors based on FLOPS weight prediction.

\begin{tabular}{|c|r|r|r|}
\hline $\begin{array}{c}\text { Model } \\
\text { Variables }\end{array}$ & $\begin{array}{c}\text { Average Error } \\
(\%)\end{array}$ & $\begin{array}{c}\text { RMS Error } \\
(\%)\end{array}$ & $\begin{array}{c}\text { Maximum Error } \\
(\%)\end{array}$ \\
\hline \hline \multicolumn{4}{|c|}{ Exclude Geometrically Infeasible Designs } \\
\hline$x_{i}$ & 13.22 & 49.49 & 5,532 \\
\hline $\ln \left(x_{i}\right)$ & 2.458 & 6.762 & 459.1 \\
\hline \hline \multicolumn{4}{|c|}{ Exclude Geometrically Unreasonable Designs } \\
\hline$x_{i}$ & 0.5249 & 1.009 & 16.97 \\
\hline $\ln \left(x_{i}\right)$ & 0.3624 & 0.6358 & 14.73 \\
\hline \hline \multicolumn{4}{|c|}{ Exclude Designs with Insufficient Range } \\
\hline$x_{i}$ & 0.3541 & 0.6153 & 11.53 \\
\hline $\ln \left(x_{i}\right)$ & 0.2727 & 0.4685 & 14.73 \\
\hline
\end{tabular}

some of the features illustrated in Figure 5.1. The minimum fuselage radius is set to $4.0 \mathrm{ft}$ along the entire length of the wing root chord. In addition, the fuselage must enclose a cone with its base, having a radius of $4.0 \mathrm{ft}$, at the leading edge of the root chord and its apex at the nose of the aircraft. A similar cone has to fit within the fuselage aft of the root chord's trailing-edge.

The majority of the new criteria, however, are based on the wing planform. Limits are placed on both the minimum and maximum allowable aspect ratio $A R$ and wing planform area $S_{w}$. Leading and trailing-edge sweep angles, $\Lambda_{l e}$ and $\Lambda_{t e}$, are prevented from assuming improbable values. Local taper ratios $c_{y_{i+1}} / c_{y_{i}}$ must be less than 1.0. Finally, the wing planform is further constrained from having a forward swept leading edge, or from having an outboard leading edge sweep greater than the inboard leading edge sweep. Applying the new criteria causes 6,473 points to be moved towards the baseline design. Figure 5.2 shows two designs which appear after the implementation of these criteria.

As a final step, a criterion is added based on aerodynamic analysis to exclude unreasonable designs that cannot be identified with geometric conditions. The approximate range is evaluated for each design using simple methods to estimate total drag on the aircraft [3]. Because of the nature of this calculation, designs having an approximate range greater than 5,000 $\mathrm{n} \mathrm{mi}$. are considered reasonable. Recall that 
the final HSCT design must attain a range of at least 5,500 $\mathrm{n} \mathrm{mi}$. This criterion moves 4,336 designs towards the baseline configuration. Figure 5.3 shows two designs which appear after the application of this final criterion.

After each new criteria is applied, a sample response surface is constructed based on the wing bending material weight estimate given by FLOPS. The response surface is created using all 19,651 designs and the associated error is measured using the same designs (Table 5.2). As the design space shrinks, the accuracy of the response surface improves significantly. In addition, the advantage of using the model with logarithms of the variables versus a polynomial model in the $x_{i}$ diminishes.

Although the response surfaces summarized in Table 5.2 are based on FLOPS estimates for wing bending material weight, the final response surfaces in this work will be constructed using data from structural optimization. Here, FLOPS is used as an inexpensive means for quantifying the reduction in error associated with reducing the design space.

\subsection{Selection of Intervening Variables}

Using the initial twenty-five variables and the 19,651 design points found after reducing the design space, a response surface model is fit to wing bending material weight estimates from FLOPS. The errors are calculated from the difference between the response surface prediction for the wing bending material weight and the value predicted by FLOPS.

Although the results given in Table 5.2 are quite good, the number of terms in the response surface function is 351 . Recall that at least $1.5 n$ function evaluations are required to produce an accurate response surface. Therefore, the number of structural optimizations necessary is at least 527 and perhaps several times larger.

A significant reduction in the size of the model function can be obtained by replacing the twenty-five design variables with a smaller set of variables that are more appropriate for a weight analysis. To maintain compatibility with prior work, these variables are also entirely dependent on the design variables. Such variables are called intervening variables in the structural optimization literature and the terminology is 
Table 5.3: Accuracy of various RS models based on FLOPS weight prediction.

\begin{tabular}{|c|c|c|c|}
\hline $\begin{array}{c}\text { Model } \\
\text { Variables }\end{array}$ & $\begin{array}{c}\text { Average Error } \\
(\%)\end{array}$ & $\begin{array}{c}\text { RMS Error } \\
(\%)\end{array}$ & $\begin{array}{c}\text { Maximum Error } \\
(\%)\end{array}$ \\
\hline \hline \multicolumn{5}{|c|}{ Linear in 25 Design Variables } \\
\hline$x_{i}$ & 3.032 & 4.868 & 67.10 \\
\hline $\ln \left(x_{i}\right)$ & 1.963 & 3.529 & 56.59 \\
\hline \hline \multicolumn{5}{|c|}{ Quadratic in 25 Design Variables } \\
\hline$x_{i}$ & 0.3541 & 0.6153 & 11.53 \\
\hline $\ln \left(x_{i}\right)$ & 0.2727 & 0.4685 & 14.73 \\
\hline \hline \multicolumn{5}{|c|}{ Linear in 10 Intervening Variables } \\
\hline$x_{i}$ & 0.1895 & 0.2932 & 2.232 \\
\hline $\ln \left(x_{i}\right)$ & 0.0781 & 0.1391 & 2.253 \\
\hline \hline \multicolumn{5}{|c|}{ Quadratic in 10 Intervening Variables } \\
\hline$x_{i}$ & 0.0025 & 0.0056 & 0.1525 \\
\hline $\ln \left(x_{i}\right)$ & 0.0062 & 0.0124 & 0.5200 \\
\hline \multicolumn{5}{c}{} \\
\hline
\end{tabular}

used here.

Returning to the description of the FLOPS weight function, the wing weight is based on a set of ten basic parameters, which are listed in Table 4.1. Each parameter can be found using the twenty-five HSCT design variables that contribute to the bending material weight. Using these parameters as the intervening variables reduces the number of terms in the model function from 351 to 66 . In addition, the accuracy of the model function actually increases when the intervening variables are introduced. A further improvement in accuracy is realized when (2.1) is used for the model function in place of (5.2). Table 5.3 summarizes these results. Note that a linear approximation in the intervening variables is more accurate than a quadratic approximation in the original variables. 


\subsection{Regression Analysis and ANOVA}

Continuing with the 19,651 HSCT designs and the FLOPS estimates for the wing bending material weight, regression analysis and analysis of variance (ANOVA) methods are used to identify unnecessary terms in each model function being considered. The intent is not to remove terms from the model functions before performing structural optimization, but simply to study the effect of removing terms. Removing terms based the FLOPS weight estimates may eliminate terms that, while not important for representing the FLOPS estimates, may be influential in modeling the structural optimization data.

Regression analysis and ANOVA are carried out separately for the model functions based on both the twenty-five design variables and the ten intervening variables. The term with the highest coefficient of variation (see Appendix A) is then removed from the model function, and the process of regression analysis and ANOVA is repeated. This sequence of operations is known as one-step-backward elimination [6].

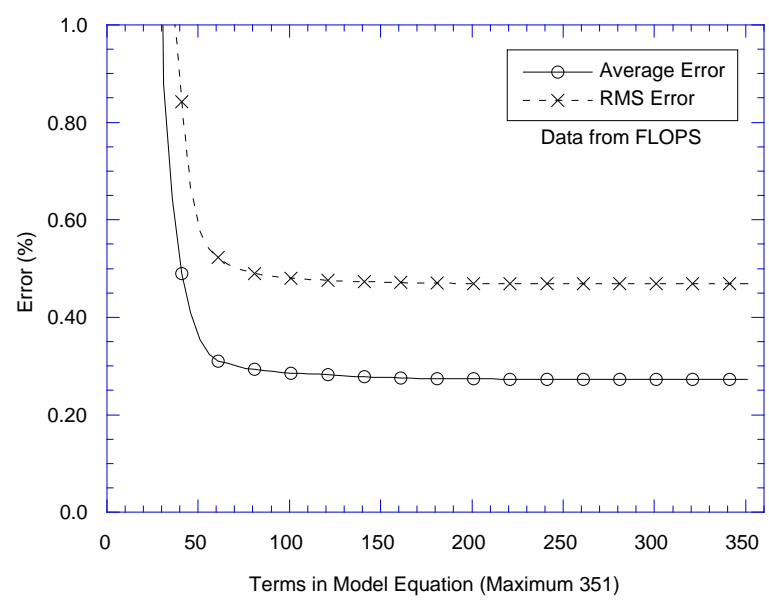

Figure 5.4: RS error versus terms remaining in design variable model function.

Figures 5.4 and 5.5 show the accuracy of the response surface compared to the number of terms remaining in the model function. Note that (5.2) is used for the twenty-five design variable case, while (2.1) is used for the ten intervening variable case. 250 terms can be removed from the design variable model function and 26 terms can be removed from the intervening variable model function without significantly 


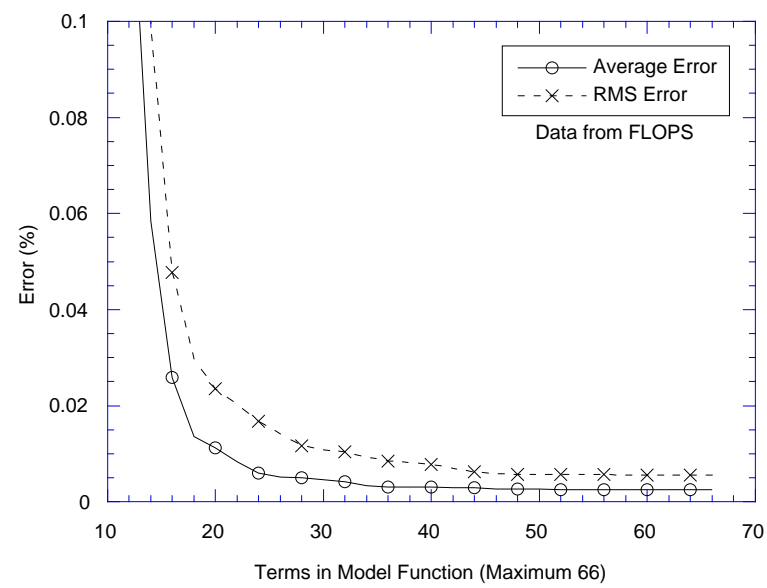

Figure 5.5: RS error versus terms remaining in intervening variable model function.

affecting accuracy. A similar situation can be expected for the structural optimization data, albeit with different model terms removed. 


\section{Chapter 6}

\section{Structural Optimization}

Within the HSCT configuration design process, estimation of wing structural weight using finite-element-based structural optimization has proven to be more accurate than estimates produced by typical weight functions. For this reason, a response surface representation of structural optimization results is developed to replace FLOPS estimates for wing bending material weight.

Due to the large number of optimizations that must be performed to create a response surface, a relatively simple structural optimization model is used. For each HSCT design, a fixed arrangement of spars and ribs is generated prior to the optimization while the skin panel thicknesses, spar areas, and rib cap areas appear as design variables. The aircraft is assumed to be built of titanium. Constraints are applied based on Von Mises stress values for each panel, spar, and rib cap element. In addition, local buckling constraints are applied. Even though bending material weight is used for the response surface, the entire wing structural weight is optimized $[26,27]$.

\subsection{Structural Optimization Model}

Because of the large number of designs which are optimized, a special mesh generator is implemented to automatically create a finite element model based on the twentyeight HSCT design variables (Table 3.1). In addition to design variables, the number 
of frames in the fuselage, the number of spars and ribs in the wing, and the chord fractions taken by the leading and trailing-edge control surfaces are specified for each design. The mesh generator creates the finite element nodes and element topology data, estimates the location of nonstructural weights, and predicts the geometry of the wing fuel tanks $[26,27]$. Fuel is assumed to be stored in thirty-one tanks throughout the aircraft.

Due to symmetry, only half of the aircraft is modeled. A typical finite element model is made up of 963 elements joined at 193 nodes with 1032 total degrees of freedom, Figure 6.1. The wing and fuselage skin are modeled by membrane elements, the spar and rib cap elements are modeled by rod elements, and the spar and rib webs are modeled by vertical rods. Initial values for the structural optimization design variables are produced using weight estimates provided by FLOPS.

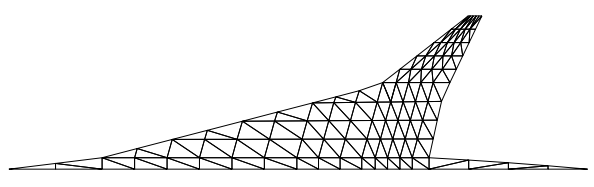

Figure 6.1: Typical structural optimization model.

The loads applied to the structural model are composed of the aerodynamic and inertia forces. Inertia loads represent the combined effects of non-structural items, fuel weight, and the distributed weight of the structure. Aerodynamic loads for supersonic flight conditions are determined using a supersonic panel method, and loads for subsonic flight conditions are from a vortex-lattice method. The structure is assumed to be rigid for the determination of aerodynamic forces. Previous studies indicated that structural flexibility did not have a large effect on the loads for this particular configuration $[14,28]$. For each design, orientation of the aerodynamic loads is governed by camber distributions generated by Carlson's program WINGDES [29]. A surface spline interpolation method is used to translate forces between aerodynamic node and structural node locations. More details about the five load cases used can be found in [30].

While acceptable for most HSCT configurations, in some cases the simplified finite 
element model coupled with a limited number of loading conditions can lead to poor structural weight predictions. For this reason, structural optimization results which differ significantly from FLOPS must be regarded with caution.

\subsection{Noise in Structural Optimization}

Structural optimization is more accurate than weight function estimates; however, they do not produce smooth functions with respect to the design variables. This is one of several reasons why structural optimization is not integrated directly into the overall configuration optimization of the HSCT. The non-smooth characteristics of structural optimization can be attributed to numerical noise associated with various parts of the optimization process as well as the irregularly shaped design space from which the optimal structure is found.

In this study, output from structural optimization is used as the observed function values for a response surface in wing bending material weight. Thus, understanding the nature of any noise produced during the structural design is critical to evaluating the performance of the response surface. To this end, twenty feasible HSCT designs are found along a line segment centered at the baseline configuration (Table 3.1). Figure 6.2 depicts both the FLOPS weight estimate and those produced by structural optimization.

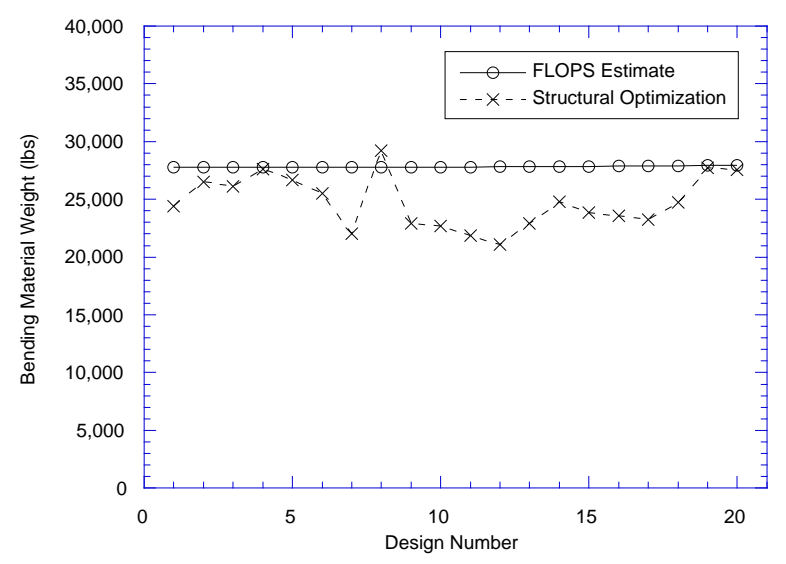

Figure 6.2: Comparison of FLOPS with structural optimization results. 


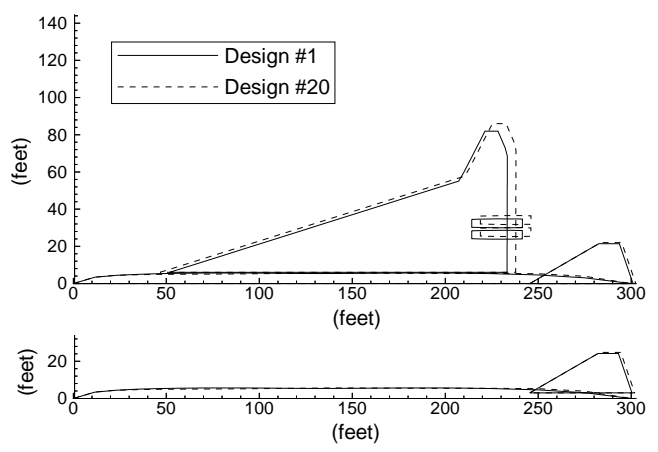

Figure 6.3: Comparison of extremum designs used for noise analysis.

Figure 6.3 shows the two extreme designs at the ends of the line segment. All twenty designs are nearly identical in planform shape, and FLOPS predicts that the wing bending material weight varies smoothly from 27,772 lbs to 27,962 lbs, a difference of only $190 \mathrm{lbs}$. While the structural optimization results are fairly smooth along portions of the line, the weight varies between 21,062 lbs and 27,712 lbs with a spike of almost $30 \%$ at the eighth design point.

Several avenues are considered to reduce the noise in the structural optimization. First, parameters which govern the optimizer are altered to promote convergence to an optimum. Initial move limits are reduced from 30\% to $10 \%$ and the stopping convergence criterion is changed from $1 \%$ to $0.1 \%$. Although this has a favorable impact on the noise, it is negligible compared to the large fluctuation at the eighth design point. Attention is next turned towards the objective function of the optimizer. Although wing bending material weight values are used for the response surface, the optimizer uses the weight of the entire wing as its objective function. For this reason, changes are made to the finite element model so that the optimizer manipulates only those portions of the wing which were accounted for in the bending material weight. Together, the changes to the optimizer and and the finite element model reduce the spike in Figure 6.3 from $30 \%$ to $27 \%$.

From the aforementioned results, it appears that the noise is not produced within the structural optimization process. Thus, attention is shifted to the loading data produced by the aerodynamic analysis. Optimizing each design using identical load data completely eliminates the spike at the eighth design point. Further, an analysis 
of the center of pressure locations based on the aerodynamic loading data for each design reveals a pattern of noise similar to that found in Figure 6.2. Thus, the most significant noise source is not the structural optimization, but instead the load generation process.

Because of time limitations associated with this study, the noise source within the load generation process has not been thoroughly investigated; however, a complete analysis of the various noise sources will appear in [26]. For this work, the structural optimization results are expected to exhibit properties similar to those depicted in Figure 6.2. Considering the distribution of data, it is clear that a quadratic polynomial can model the FLOPS data with little or no error. In contrast, errors of as much as $27 \%$ can be anticipated if a similar polynomial is used to model the structural optimization data.

\subsection{Parallelized Structural Optimization}

Because of the numerous structural optimizations required for the response surface, this problem is well suited for coarse grain parallel computation. Coarse grain parallelization implies that structural optimizations for different HSCT designs are performed simultaneously on separate processors. Each processor maintains its own data, so that interaction between the processors is not necessary.

As the number of processors is increased on a distributed memory architecture machine like the Intel Paragon, disk I/O may become a bottleneck limiting the efficiency of the parallel computations. This fact is the primary reason for choosing the GENESIS structural optimization code for implementation in the parallel environment. GENESIS, a code developed and supported by Vanderplaats, Miura and Associates, Inc [19] is available from the developer in a reduced I/O form making it an effective code to use on the Paragon.

The speedups of a parallel computation is defined as $T_{s} / T_{p}$ where $T_{s}$ is the serial execution time and $T_{p}$ is the parallel execution time using $p$ processors. In an ideal situation, speedup would be equal to the number $p$ of processors being used. Figure 6.4 shows the benefits of the reduced I/O version of GENESIS. With the standard 
version, maximum speedup levels off at 2.3, regardless of the number of processors, while the reduced I/O version achieves a speedup of 11.7 using 20 processors. This is still rather poor, showing how ill-suited packages developed for serial computation, like GENESIS, are for parallel computation.

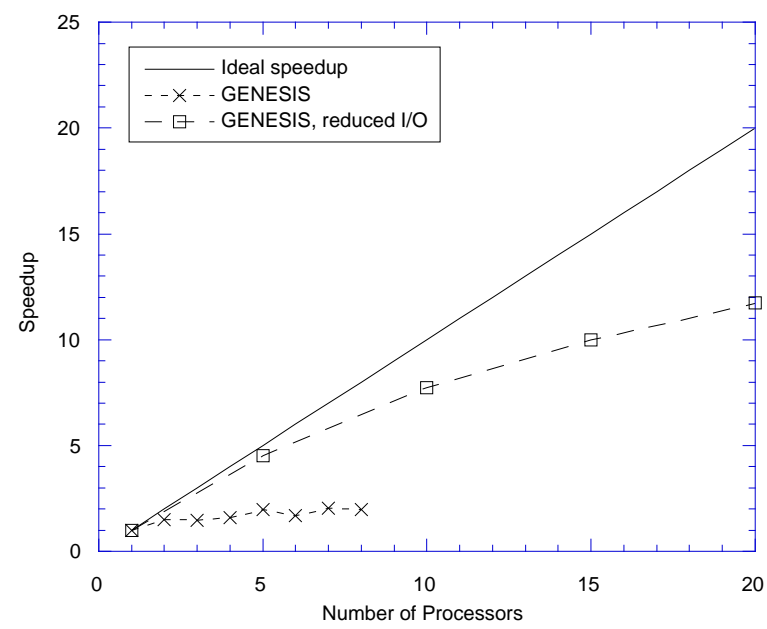

Figure 6.4: Ideal versus actual speedup for parallel execution of the GENESIS. 


\section{Chapter 7}

\section{RSM for Structural Optimization}

This section concerns the creation of a response surface for the wing bending material weight based on data from the structural optimization code GENESIS. As discussed in the last section, there is significant numerical noise within the structural optimization process, perhaps as much as $27 \%$. It is thus difficult to separate noise in the optimized wing bending material weights from fitting error, and thus the apparent error in the response surface approximation must be interpreted properly. Indeed, a substantial part of the error may represent a desirable smoothing of the results from the structural optimization.

With the FLOPS weight function, all 19,651 configurations could be used to construct a response surface. However, the expense of structural optimization limits the number of designs which can be considered to a small subset of the 19,651 points. $D$ optimality [18] provides a rational criterion for choosing this subset. For an $n$-term model function, the cost of assessing the merit of each candidate design is $\mathcal{O}\left(n^{3}\right)$. Typically, $D$-optimal point sets are computed by simple exchange algorithms or genetic algorithms. Unfortunately, it is computationally infeasible for either class of algorithms to work with the 351-term polynomial in the twenty-five design variables at all 19,651 points. The cost of evaluating each merit function is simply too great.

Candidate HSCT configurations are chosen by picking 3,000 points (mostly at random, but also containing previously analyzed designs) from the 19,651 points; the condition number of the least squares matrix $\mathbf{X}$, cond $\mathbf{X}$, (Appendix A) for these 3,000 
points is below $10^{4}$. Although the set of 3,000 candidate designs is just a fraction of the 19,651, it is still infeasible to use any of the available algorithms for calculating $D$-optimal point sets. As before, the cost of evaluating each merit function is simply too great.

An ad hoc approach is to decompose the polynomial model into a sum of polynomials with fewer terms, find a $D$-optimal set for each summand, and then take the union of all these $D$-optimal sets. Not just any decomposition will yield a well conditioned $\mathbf{X}$ matrix for the union (in particular a decomposition into 7 disjoint sets of summands does not work). Using seven summands, each of which contained all the linear terms and 50 quadratic terms (25 quadratic terms in the seventh summand), and finding $150 \mathrm{D}$-optimal points for each summand (125 D-optimal points for the last one), yields 1,025 designs, for which cond $\mathbf{X}=5.7 \times 10^{3}$.

Structural optimizations with GENESIS [19] are performed for each of the 1,025 designs to find the optimum wing bending material weight. 991 of the optimizations are successful and cond $\mathbf{X}=5.7 \times 10^{3}$. Using the same data points and the 66 -term model function in the intervening variables, the condition number of $\mathbf{X}=5.9 \times 10^{4}$. These 991 points, referred to as Set A, are used to create the response surfaces for bending material weight.

A similar procedure is used to identify another set of 971 points (Set B), used for checking the accuracy of the response surface created by Set A. Taking results from both Sets A and B, the ratios of structural optimization values to values predicted by FLOPS are in the range of 0.51 to 2.05 , and the average value is 0.89 .

Using every design in Set A, two response surfaces are created, one using the 66term model function in the intervening variables and the other using the 351-term model function in the design variables. Again, the program RSG is used to create and evaluate each response surface (Appendix F). To determine what portion of Set A is necessary to accurately model the entire design space, a number of $D$-optimal sets are found from within that set. For each $D$-optimal subset of Set A, a response surface is created using the associated model function and then evaluated using Set B. The relationship between the error and the number of points used to create the response surface is depicted in Figure 7.1. From the figure it appears that the intervening 
variables require 300-400 points for the average error to stabilize, while for the design variables, 1,000 points may not be enough.

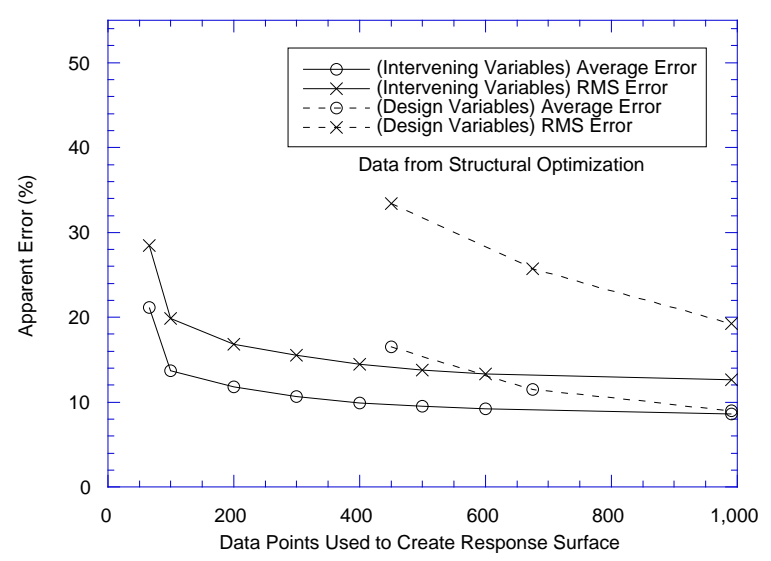

Figure 7.1: Apparent accuracy of full RS in design and intervening variables.

Returning to the ratio of the number of analysis points to the number of terms in the model function discussed earlier, for the full model function in the intervening variables this ratio appears to be $300 / 66 \approx 4.5$. The ratio for the full design variable model function appears to be at least $991 / 351 \approx 2.8$ and most likely much higher. Recall that a smaller value, 1.5, was predicted using the six-term polynomial in two dimensions.

ANOVA and regression analysis with data from Set A are used to remove unnecessary terms from the 66-term model in the ten intervening variables and the 351-term model in the twenty-five design variables. Results are plotted in Figures 7.2 and 7.3, which are similar to those found using the FLOPS estimates for wing bending material weight (Figures 5.4 and 5.5). The 66-term intervening variable model function can be reduced to 15 terms and the 351-term design variable model function to 61 terms without adversely affecting the approximation.

The motivation for using ANOVA and regression analysis is to reduce the incidence of large errors in the response surface formulations. However, Figures 7.2 and 7.3 cannot demonstrate this effect because the same data Set A is used to both create and evaluate each response surface. For this reason, apparent error is evaluted using Set B for identical response surfaces and the results are given in Figures 7.4 and 7.5. 


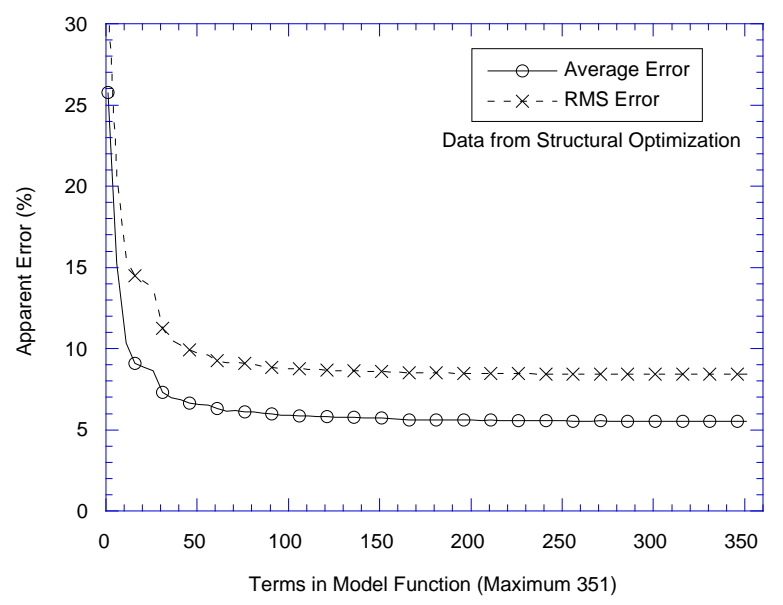

Figure 7.2: RS apparent error versus terms in design variable model function.

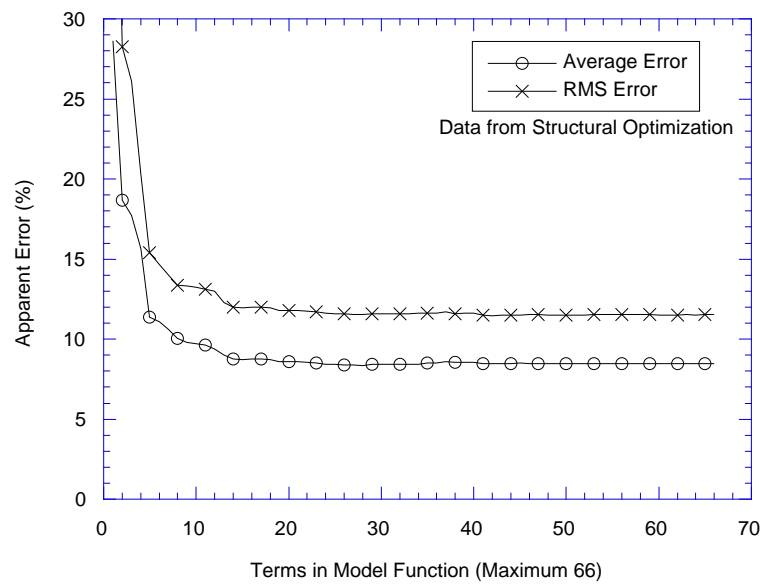

Figure 7.3: RS apparent error versus terms in intervening variable model function. 
Removing unnecessary model function terms significantly reduces both average error and RMS error for the design variable response surface. A similar, albeit smaller, reduction also occurs for the intervening variable model function. Again, it appears that the intervening variable model function should be reduced to 15 terms and the design variable model function should be reduced to 61 terms.

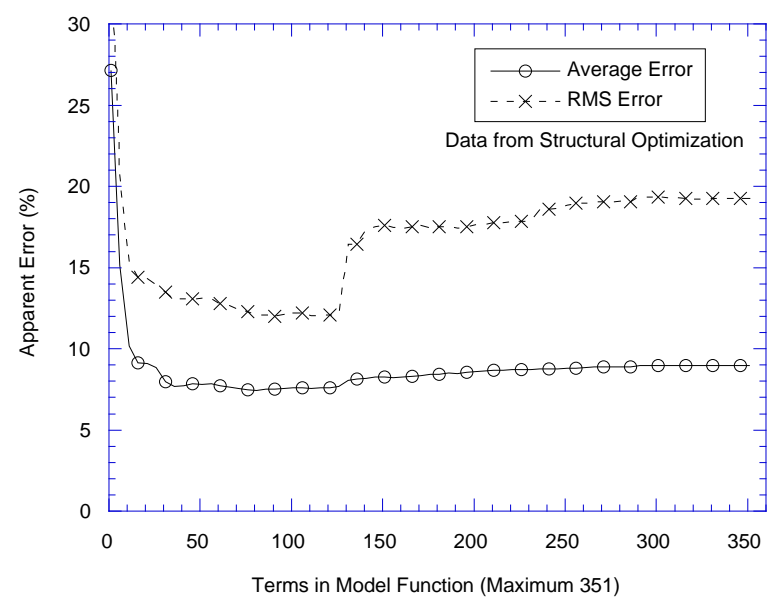

Figure 7.4: Reduced apparent error using ANOVA and design variable RS.

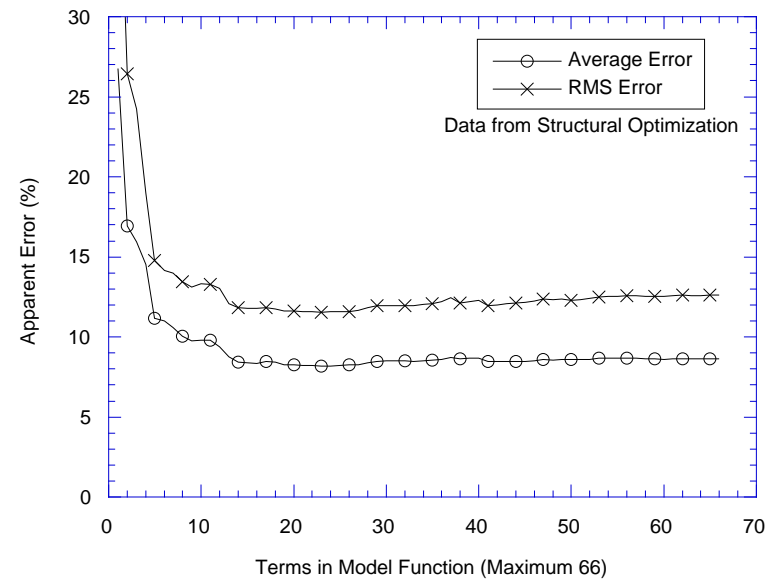

Figure 7.5: Reduced apparent error using ANOVA and intervening variable RS.

These reduced term polynomials are now used to determine the number of points required to produce a response surface that accurately reflects wing bending material weight over the entire design space. Referring to Figure 7.6, for each number $k$ of 
data points and both reduced term models, a $k$ point $D$-optimal subset of Set A is found and used to compute a response surface, whose quality is measured over Set B. For both the intervening variables and the design variables, Figure 7.6 indicates that 200-250 points are needed for the RMS error to stabilize. For the design variables, this translates into a ratio of the number of analysis points to the number of terms in the model function of $200 / 61 \approx 3.3$, and $200 / 15 \approx 13.3$ for the intervening variables.

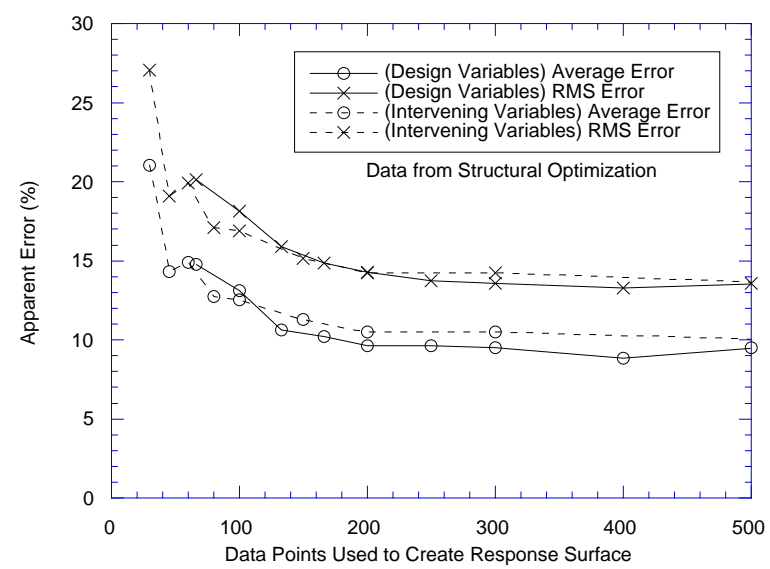

Figure 7.6: Apparent accuracy of reduced RS in design and intervening variables.

The response surface created using the 61-term polynomial in the design variables will henceforth be referred to as RS1, and RS2refers to the 15-term polynomial in the intervening variables. RS3is the response surface using

$$
y=c_{0}+c_{1} W_{b_{F}}
$$

as the model, essentially, a linear function in the FLOPS prediction of the bending material weight. Apparent accuracies for RS1, RS2, and RS3, are summarized in Table 7.1. $R S 1_{\mathrm{A}}, \mathrm{RS} 2_{\mathrm{A}}$, and $\mathrm{RS} 3_{\mathrm{A}}$ are created using Set $\mathrm{A}$. The union of Set $\mathrm{A}$ and Set $\mathrm{B}$ are used to create $\mathrm{RS} 1_{\mathrm{AB}}, \mathrm{RS} 2_{\mathrm{AB}}$, and $\mathrm{RS} 3_{\mathrm{AB}}$. In each case, only Set $\mathrm{B}$ is used to evaluate the response response surface. A detailed description of each response surface is given in Appendix D.

Comparing results from Tables 5.3 and 7.1 highlights the decrease in response surface accuracy accompanying a move from the FLOPS data to the GENESIS data. Focusing on the intervening variables, one could postulate that the variables are only 
Table 7.1: Apparent accuracy of various RS models based on GENESIS weight prediction.

\begin{tabular}{|l|r|r|r|}
\hline Name & $\begin{array}{c}\text { Average Error } \\
(\%)\end{array}$ & $\begin{array}{c}\text { RMS Error } \\
(\%)\end{array}$ & $\begin{array}{c}\text { Maximum Error } \\
(\%)\end{array}$ \\
\hline \hline \multicolumn{5}{|c|}{ Generate RS Using Set A } \\
\hline $\mathrm{RS}_{\mathrm{A}}$ & 7.72 & 12.78 & 114.58 \\
\hline $\mathrm{RS} 2_{\mathrm{A}}$ & 8.84 & 12.11 & 63.67 \\
\hline $\mathrm{RS} 3_{\mathrm{A}}$ & 13.84 & 20.49 & 72.13 \\
\hline \hline \multicolumn{5}{|c|}{ Generate RS Using Set A and Set B } \\
\hline $\mathrm{RS} 1_{\mathrm{AB}}$ & 6.86 & 10.66 & 72.05 \\
\hline $\mathrm{RS} 2_{\mathrm{AB}}$ & 8.54 & 11.65 & 61.68 \\
\hline $\mathrm{RS} 3_{\mathrm{AB}}$ & 13.66 & 20.43 & 73.24 \\
\hline
\end{tabular}

suited to model the FLOPS estimates. Indeed, the ten intervening variables are straight out of the equations used within FLOPS. It is possible that terms such as $B_{z e}$ and $B_{z}$ (Appendix B) are inappropriate for modeling the structural optimization results. Still, it is reasonable to assume that the twenty-five design variables can model either the FLOPS estimates or the structural optimization results with similar accuracy. This is not the case. In fact, a strong case can be made for using the intervening variables over the design variables. Both the RMS and maximum errors associated with the RS2are less than those associated with the other two response surfaces. In addition, a close inspection of $\mathrm{RS} 1_{\mathrm{A}}$ and $\mathrm{RS} 1_{\mathrm{AB}}$ shows large differences between the corresponding coefficient values in comparison to the differences associated with the RS2 and RS3 response surfaces (Appendix D). This may reflect RS1's sensitivity to noise in the structural optimization data.

There are two plausible explanations for the results presented in Tables 5.3 and 7.1: (1) a quadratic model is inadequate for the wing bending material weight over the entire domain being considered here, or (2) the GENESIS optima are incorrect, due to noisy aerodynamic loads or some other source of noise. Chapter 6 offers evidence in support of (2), but does not rule out (1). Possibly both (1) and (2) occur.

In general, response surfaces created using the union of Set A and Set B produce apparent errors which are consistently lower than response surfaces produced with Set A. This is expected since Set B is used to evaluate all six response surfaces, but 
is only used in the creation of $\mathrm{RS} 1_{\mathrm{AB}}, \mathrm{RS} 2_{\mathrm{AB}}$, and $\mathrm{RS} 3_{\mathrm{AB}}$. In each case, however, the reduction in error is small. This indicates that Set A provides adequate information to model the design points within Set B. 


\section{Chapter 8}

\section{Response Surface Implementation}

Implementation of each response surface is accomplished by modifying the gross takeoff weight $W_{g}$ calculations within the weight module of FLOPS. In place of FLOPS estimates for wing bending material weight $W_{b_{\mathrm{F}}}$, response surface predictions $W_{b_{\mathrm{RS} 1}}$, $W_{b_{\mathrm{RS} 2}}$, and $W_{b_{\mathrm{RS} 3}}$ are used. From Chapter $7, \mathrm{RS} 1$ refers to the 61 -term polynomial in the design variables, RS2 refers to the 15-term polynomial in the intervening variables, and RS3 refers to the linear function in the FLOPS prediction of the bending material weight $W_{b_{F}}$. These predictions are intended for use in the reasonable design space and cannot be relied upon outside this region. Therefore, attempts are made to use $W_{b_{\mathrm{F}}}$ whenever information is requested outside of the reasonable design space.

With two exceptions, the criteria listed in Table 5.1 are used to identify the reasonable design space. First, the range criterion is not evaluated because of its relatively high computational expense. Second, an additional criterion is added to exclude unreasonable predictions by the response surface. Similar to the criterion on $W_{b_{\mathrm{F}}}$, if the response surface value falls outside the range $20,000 \mathrm{lbs}<W_{b_{\mathrm{RS}}}<120,000 \mathrm{lbs}$, the design is considered unreasonable.

Switching between $W_{b_{\mathrm{F}}}$ and $W_{b_{\mathrm{RS}}}$ at the edge of the reasonable design space can produced unwanted discontinuities in the bending material weight with respect to small changes in the design variables. For this reason, a smoothing function is used to ensure a continuous transition between $W_{b_{\mathrm{RS}}}$ and $W_{b_{\mathrm{F}}}$ when results from the response 
surface can no longer be relied upon. The smoothing function

$$
W_{b}= \begin{cases}W_{b_{R S}}, & r_{\max } \leq 0, \\ f\left(W_{b_{R S}}, W_{b_{F}}\right), & 0<r_{\max }<0.10, \\ W_{b_{F}}, & r_{\max } \geq 0.10,\end{cases}
$$

where

$$
f(a, b)=a\left[1-\sin ^{2}\left(5 r_{\max } \pi\right)\right]+b \sin ^{2}\left(5 r_{\max } \pi\right),
$$

is based on the maximum violation $r_{\max }$ among the aforementioned criteria which identify reasonable designs. A value $r_{\max }>0$ is indicative of a violated constraint and $r_{\text {max }}=1.0$ represents a maximum constraint violation of $100 \%$. In this work, $W_{b_{\mathrm{RS}}}$ is considered inaccurate if any constraint is violated by more than $10 \%, r_{\max }>0.10$. Equation 8.2 provides a smooth transition between $W_{b_{\mathrm{RS}}}$ and $W_{b_{\mathrm{F}}}$ and is continuous in the first derivative at $r_{\max }=0$ and $r_{\max }=0.10$.

The FLOPS gross takeoff weight $W_{g}$ calculation is an iterative process and care must be taken to guarantee convergence within a reasonable number of iterations. Without a response surface, approximately seven iterations are required for FLOPS to converge upon a solution. Response surface results are introduced into the $W_{g}$ calculations using (8.1) at each iteration. Unfortunately, $r_{\max }$ is very sensitive to fluctuations in $W_{b_{\mathrm{RS}}}$ near the edge of the reasonable design space. This can significantly reduce the rate of convergence and in some cases prevent convergence altogether. Of course, convergence can always be obtained using $W_{b_{\mathrm{F}}}$ alone; however, switching between $W_{b_{\mathrm{F}}}$ and $W_{b_{\mathrm{RS}}}$ from one design to the next creates noise in the HSCT objective function and prevents successful HSCT design optimization.

Implementation of (8.1) is further complicated when using RS2 and RS3. Unlike RS1, RS2 and RS3 use outputs from FLOPS as input variables. Intermediate outputs from FLOPS are often representative of unreasonable designs. In fact, the first iteration of FLOPS assumes that $W_{g}$ is zero. In general, it is impossible to evaluate the reasonability of intermediate designs because the associated design variables are unknown.

Considering the aforementioned issues, a method for calculating the gross takeoff weight $W_{g}$ is implemented based on achieving the following goals. First, for a reasonable design, a solution using $W_{b_{\mathrm{RS}}}$ is always preferred over one which uses $W_{b_{\mathrm{F}}}$. 
Next, during the iterative solution for $W_{g}$, switching between $W_{b_{\mathrm{F}}}$ and $W_{b_{\mathrm{RS}}}$ must be avoided to maximize the rate of convergence. The method cannot fail or take an unreasonable amount of time to converge. Finally, values for $W_{b_{\mathrm{RS}}}$ should not be utilized for unreasonable designs.

These goals are accomplished by introducing an artificial constraint violation which is considered along with the other constraints previously discussed in this chapter during the calculation of $r_{\max }$. The new constraint is defined as

$$
r_{a}= \begin{cases}0.10\left(1-\frac{n_{F}+n_{0}}{n_{c}}\right) & n_{F}+n_{0} \leq n_{c} \\ 0 & n_{F}+n_{0}>n_{c}\end{cases}
$$

where $n_{F}$ is the current iteration number within FLOPS. The terms $n_{c}$ and $n_{0}$ are constants chosen to maximize the rate of convergence. In conjunction with (8.1), $r_{a}$ provides a smooth transition between $W_{b_{\mathrm{F}}}$ and $W_{b_{\mathrm{RS}}}$ at the edge of the reasonable design space. For sufficiently large $n_{c}$ and $n_{0}=0, W_{b_{\mathrm{RS}}}$ is essentially ignored during the initial iterations when outputs of FLOPS are representative of unreasonable designs. As $\left(n_{F}+n_{0}\right)$ approaches $n_{c}$, the smoothing function (8.1) is be dominated by the actual constraint violations.

Within the reasonable design space, the artificial constraint violation allows for rapid convergence on a gross takeoff weight. For $n_{c}=5$ and $n_{0}=0$, convergence is normally achieved within 12 iterations. At the edge of the reasonable design space, larger values of $n_{c}$ generally produce better results. Since the majority of weight calculations are done on reasonable designs, initial values for $n_{c}$ and $n_{0}$ are set to 5 and 0 respectively. If convergence is not achieved within $\left(n_{c}-n_{0}+10\right)$ iterations or $r_{\max }$ becomes greater than $0.10, n_{F}$ is reset to zero, $n_{c}$ is raised by $10, n_{0}$ is set to $n_{c} / 2$ using the new value of $n_{c}$, and the iteration process is repeated beginning with the most recent intermediate values generated by FLOPS. If the total number of iterations exceeds 180, an extremely rare occurrence, then $W_{b_{\mathrm{F}}}$ is used unconditionally. Convergence is always achieved within 200 cycles.

With one exception, this method achieves each of the defined goals. Problems arise, however, when $W_{b_{\mathrm{RS}}}$ falls slightly below 20,000 lbs. Here, the constraint based on 20,000 lbs $<W_{b_{\mathrm{RS}}}<120,000 \mathrm{lbs}$ is very sensitive to small changes in $W_{b_{\mathrm{RS}}}$ and rapid changes in $r_{\max }$ reduce the likelihood of convergence. This problem is addressed 
by multiplying the constraint based on $W_{b_{\mathrm{RS}}}$ by 0.20 before considering it in the calculation of $r_{\max }$. Thus, $W_{b_{\mathrm{RS}}}$ is allowed to assume values as low as 10,000 lbs before the constraint violation exceeds 0.10. Experience shows that even with the constraint relaxation, the optimizer rarely pursues HSCT designs where $W_{b_{\mathrm{RS}}}<18,000 \mathrm{lbs}$.

The need to relax the constraint on $W_{b_{\mathrm{RS}}}$ may be indicative of a poor assumption made during this study. Values for $W_{b}$ less than 20,000 lbs are considered unreasonable throughout this work. However, this assumption is based on results from previous studies where optimal HSCT designs were significantly heavier [3]. Recent modifications and improvements to the analysis methods have significantly lowered $W_{g}$ for optimal designs. Perhaps a better constraint on $W_{b}$ would have been 15,000 lbs $<W_{b}<60,000$. 


\section{Chapter 9}

\section{HSCT Design Optimization}

Complete HSCT design optimizations are performed to evaluate the response surface approximations to wing bending material weight $W_{b}$. Results are then compared with the GENESIS prediction for wing bending material weight $W_{b_{\mathrm{G}}}$. Seven optimizations are completed, each beginning from the baseline design detailed in Table 3.1. During the first optimization, the FLOPS wing bending material weight $W_{b_{\mathrm{F}}}$ is used to determine the values of the design variables which minimize the takeoff gross weight $W_{g}$. The remaining six optimizations are performed with $\mathrm{RS} 1_{\mathrm{A}}, \mathrm{RS} 1_{\mathrm{AB}}, \mathrm{RS} 2_{\mathrm{A}}, \mathrm{RS} 2_{\mathrm{AB}}$, $\mathrm{RS} 3_{\mathrm{A}}$, and $\mathrm{RS} 3_{\mathrm{AB}}$ respectively, in conjunction with the smoothing function (8.1).

Recall from Chapter 7 that RS1 refers to the 61-term polynomial in the design variables, RS2 refers to the 15-term polynomial in the intervening variables, and RS3 refers to the linear function in the FLOPS prediction of the bending material weight $W_{b_{\mathrm{F}}}$. The subscripts $\mathrm{A}$ and $\mathrm{AB}$ indicate which data set is used to generate the specific response surface. Set A is used for all response surfaces having the subscript A, while the union of Set A and Set B is used to generate the response surfaces with the subscript AB.

Figure 9.1 and Table 9.1 give the gross takeoff weight $W_{g}$ for each design as well as the error in the bending material weight value $W_{b}$ used for each optimal design. Here, the error is based on a comparison with the bending material weight predicted by GENESIS $W_{b_{\mathrm{G}}}$. More detailed results from each optimization are given in Tables 9.3 through 9.5, the HSCT planforms are plotted in Figures 9.2 through 9.4, and a 
complete summary of the design variables for each configuration is given in Appendix E.

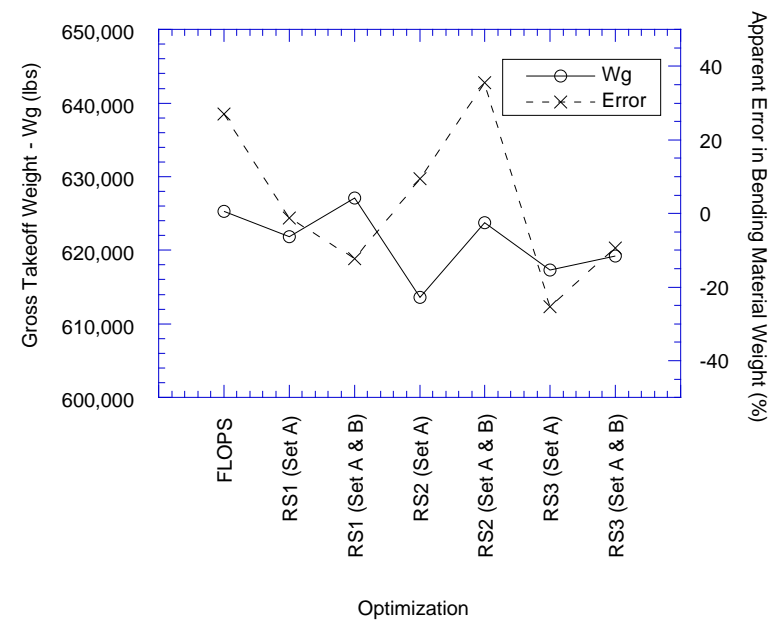

Figure 9.1: Comparison of $W_{g}$ and $W_{b}$ for each optimal HSCT design.

Table 9.1: Numerical comparison of $W_{g}$ and $W_{b}$ for each optimal HSCT design.

\begin{tabular}{|l|c|c|c|r|}
\hline $\begin{array}{c}\text { Optimization } \\
\downarrow\end{array}$ & $\begin{array}{c}W_{g} \\
(\mathrm{lbs})\end{array}$ & $\begin{array}{c}W_{b} \\
(\mathrm{lbs})\end{array}$ & $\begin{array}{c}W_{b_{\mathrm{G}}} \\
(\mathrm{lbs})\end{array}$ & $\begin{array}{c}\text { Error } \\
(\%)\end{array}$ \\
\hline \hline FLOPS & 625,325 & 23,807 & 18,753 & 26.95 \\
\hline RS1 $_{\mathrm{A}}$ & 621,833 & 22,688 & 22,988 & -1.31 \\
\hline RS1 $_{\mathrm{AB}}$ & 627,119 & 20,723 & 23,641 & -12.34 \\
\hline RS2 $_{\mathrm{A}}$ & 613,601 & 18,078 & 16,514 & 9.47 \\
\hline RS2 $_{\mathrm{AB}}$ & 623,767 & 19,894 & 14,681 & 35.51 \\
\hline RS3 $_{\mathrm{A}}$ & 617,309 & 21,706 & 29,083 & -25.37 \\
\hline RS3 $_{\mathrm{AB}}$ & 619,245 & 20,083 & 22,164 & -9.39 \\
\hline
\end{tabular}

Optimizations using RS1 produce results which may appear misleading due to the properties of the smoothing function (8.1). From Table 9.3 the optimization completed using $\mathrm{RS} 1_{\mathrm{A}}$ gives $W_{\mathrm{RSS}_{\mathrm{A}}}=238,720 \mathrm{lbs}$; however, the optimized $W_{b}$ is given as 22,688 lbs. Here, $W_{b_{\mathrm{RS} 1_{\mathrm{A}}}}$ is well above the constraint limit 20,000 lbs $<W_{b}<$ 120,000 lbs for reasonable bending material weight values. Therefore, the HSCT analysis code uses $W_{b_{\mathrm{F}}}$ in place of $W_{b_{\mathrm{RS} 1_{\mathrm{A}}}}$ for this particular design. A similar situation occurs for the optimization using $\mathrm{RS} 1_{\mathrm{AB}}$ where $W_{b_{\mathrm{RS} 1_{\mathrm{AB}}}}=207,234$. Thus, optimal 
HSCT designs generated using RS1 are based on FLOPS values for the bending material weight instead of predictions from the response surface.

The gross takeoff weight $W_{g}$ for each design is given in Figure 9.1. The optimal design obtained using $\mathrm{RS}_{\mathrm{AB}}$ produces the heaviest design while $\mathrm{RS} 2_{\mathrm{A}}$ produces the lightest design; although, the difference between the two designs is only 13,518 lbs or about $2 \%$ of $W_{g}$. In addition to values for $W_{g}$, Table 9.1 also gives the apparent accuracy of the bending material weight value $W_{b}$ used during the optimization with respect to structural optimization $W_{b_{\mathrm{G}}}$. The smallest absolute apparent error, $1.31 \%$, is obtained using $\mathrm{RS}_{\mathrm{A}}$; however, this may again appear misleading. Remember that $W_{b_{\mathrm{F}}}$ is used for the final design because $W_{b_{\mathrm{RS} 1_{\mathrm{A}}}}$ falls outside of the reasonable limits for bending material weight. The largest apparent error in $W_{b}, 35.51 \%$, is associated with the optimization using $\mathrm{RS} 2_{\mathrm{AB}}$.

A more useful comparison of response surface accuracies is given in Table 9.2. Values for $W_{b}$ are computed using all six response surfaces and FLOPS at each of the seven optimal designs. The apparent accuracy of each method is then measured in comparison to the GENESIS prediction $W_{b_{\mathrm{G}}}$ and presented in the table. The first seven rows of Table 9.2 correspond to the optimal HSCT designs, while each column represents a different method for calculating wing bending material weight. The final row gives the average apparent error associated with each method. Each value which appears in boldface type is the apparent accuracy of the method used to find the corresponding optimal design. These values are identical to those found in Table 9.1. Clearly, $\mathrm{RS} 2_{\mathrm{A}}$ and $\mathrm{RS} 2_{\mathrm{AB}}$ are the most accurate with average apparent errors less than $17 \%$. In contrast, $\mathrm{RS} 1_{\mathrm{A}}$ and $\mathrm{RS} 1_{\mathrm{AB}}$ are the least accurate with average apparent errors greater than $775 \%$. FLOPS and RS3 are characterized by apparent errors near $20 \%$.

Overall, FLOPS does a better job at estimating $W_{b}$ than previous studies had suggested. This may reflect the fact that its weight function was created based on the weight of actual wings and the structural optimization weight of good aerodynamic designs. Remember that the HSCT is a new aircraft and relatively little information from actual wings is available; therefore, FLOPS must rely predominantly on structural optimization. Prior to recent improvements in the analysis methods within 
Table 9.2: Apparent error of $W_{b}$ values at each optimal HSCT design.

\begin{tabular}{|c|c|c|c|c|c|c|c|}
\hline $\begin{array}{c}\text { Optimization } \\
\downarrow\end{array}$ & $\begin{array}{l}W_{b_{\mathrm{F}}} \\
(\%)\end{array}$ & $\begin{array}{c}W_{b_{\mathrm{RS} 1_{\mathrm{A}}}} \\
(\%)\end{array}$ & $\begin{array}{c}W_{b_{\mathrm{RS} 1 \mathrm{AB}}} \\
(\%)\end{array}$ & $\begin{array}{c}W_{b_{\mathrm{RS} 2 \mathrm{~A}}} \\
(\%)\end{array}$ & $\begin{array}{c}W_{b_{\mathrm{RS} 2 \mathrm{AB}}} \\
(\%)\end{array}$ & $\begin{array}{c}W_{b_{\mathrm{RS} 3 \mathrm{~A}}} \\
(\%)\end{array}$ & $\begin{array}{c}W_{b_{\mathrm{RS} 3 \mathrm{AB}}} \\
(\%)\end{array}$ \\
\hline FLOPS & 26.95 & 351.76 & 386.11 & 19.70 & 22.68 & 16.10 & 14.00 \\
\hline $\mathrm{RS} 1_{\mathrm{A}}$ & -1.31 & 938.45 & 696.56 & -10.25 & -7.26 & -9.40 & -11.28 \\
\hline $\mathrm{RS} 1_{\mathrm{AB}}$ & 12.34 & 1048.09 & 776.59 & -9.96 & -7.23 & -11.06 & -12.85 \\
\hline $\mathrm{RS} 2_{\mathrm{A}}$ & 36.97 & 836.12 & 764.02 & 9.47 & 17.91 & 25.77 & 23.16 \\
\hline $\mathrm{RS} 2_{\mathrm{AB}}$ & 60.55 & 1053.08 & 930.15 & 26.12 & 35.51 & 46.95 & 44.21 \\
\hline $\mathrm{RS} 3_{\mathrm{A}}$ & -18.40 & 463.81 & 467.62 & -22.24 & -20.84 & -25.37 & -26.73 \\
\hline $\mathrm{RS} 3_{\mathrm{AB}}$ & 0.77 & 388.44 & 411.28 & -6.02 & -3.81 & -7.40 & -9.39 \\
\hline & \multicolumn{7}{|c|}{ Average Apparent Error (Absolute Values) } \\
\hline Value & 22.47 & 725.68 & 633.19 & 14.82 & 16.46 & 20.29 & 20.23 \\
\hline
\end{tabular}

the HSCT design code, optimal designs often exhibited less than ideal aerodynamic qualities [24]. Thus, previous studies may have utilized FLOPS in regions of design space where neither actual wings or structural optimization data was available. This may account for the poor weight estimates from FLOPS when compared to structural optimization.

The consistently poor results obtained with RS1 may be attributed to one or a combination of the following problems. First, a quadratic function in the original design variables may be a poor model for the bending material weight, a conclusion supported by the maximum apparent errors in Tables 5.3 and 7.1. Second, the larger number of terms used by RS1 may have resulted in more opportunity to fit some of the noise in the structural optimization and therefore produce poor predictions for otherwise good designs.

As expected RS2 produces the most accurate results. Recall that RS2 has the lowest maximum apparent errors in Tables 5.3 and 7.1. RS2 not only produces the least amount of apparent error, but optimal designs generated with RS2 have the lowest values for $W_{b}$. Optimizing with $\mathrm{RS} 2_{\mathrm{A}}$ yields $W_{b_{\mathrm{RS} 2_{\mathrm{A}}}}=18,078 \mathrm{lbs}$ and optimizing with $\mathrm{RS} 2_{\mathrm{AB}}$ gives $W_{b_{\mathrm{RS} 2} \mathrm{AB}}=19,894$ lbs. In addition, the optimizer does not seem to be exploiting deficiency in the response surface. Structural optimization gives $W_{b_{\mathrm{G}}}=$ $16,514 \mathrm{lbs}$ for the design generated with $\mathrm{RS} 2_{\mathrm{A}}$ and $W_{b_{\mathrm{G}}}=14,681 \mathrm{lbs}$ for the design generated with RS2 $\mathrm{AB}$. Again, these are the lowest values for $W_{b_{\mathrm{G}}}$ of the seven optimal 
designs.

Although Table 7.1 shows a consistent increase in response surface apparent accuracy when using the union of Set A and B to generate the response surface rather than just Set A, a similar trend does not necessarily appear in Table 9.2. While the apparent error in RS3 falls by less than $0.1 \%$, the apparent error in RS2 actually rises by $1.5 \%$ when the additional points from Set B are used. RS1 benefits the most from the additional points. Here, the apparent error falls from $726 \%$ to $633 \%$. The inconsistent results given in Table 9.2 can most likely be attributed to noise in the structural optimization data.

For the optimal designs produced using RS2, values for $W_{b_{\mathrm{G}}}$ are well below the 20,000 lbs which defines the lower limit of wing bending material weight values in Table 5.1. Two possible explanations may account for this discrepancy. First, values for $W_{b_{\mathrm{G}}}$ might be inaccurate, perhaps reflecting noise within the structural optimization process or limitations of the structural optimization model. Another, perhaps more plausible, explanation might be that the reasonable limits on $W_{b} 20,000 \mathrm{lbs}<$ $W_{b}<120,000 \mathrm{lbs}$, set forth in Chapter 5, are inappropriate. This is supported by the relatively low values for $W_{b_{\mathrm{F}}}$ associated with each of the seven optimal designs. The aforementioned limits on $W_{b}$ are based on previous work where optimal HSCT designs were significantly heavier [3]. A better constraint on $W_{b}$ for this study might have been $15,000 \mathrm{lbs}<W_{b}<60,000$. 
Table 9.3: Optimal HSCT designs using response surface RS1.

\begin{tabular}{|c|c|c|c|}
\hline Optimization $\rightarrow$ & FLOPS & $\mathrm{RS} 1_{\mathrm{A}}$ & $\mathrm{RS} 1_{\mathrm{AB}}$ \\
\hline \multicolumn{4}{|c|}{ Planform Geometry } \\
\hline Root chord (ft) & 154.3 & 158.9 & 159.8 \\
\hline Tip chord (ft) & 7.53 & 7.59 & 8.22 \\
\hline Aspect Ratio & 1.77 & 1.63 & 1.73 \\
\hline Wing Area $\left(\mathrm{ft}^{2}\right)$ & 10,184 & 10,056 & 10,246 \\
\hline \multicolumn{4}{|c|}{ Performance Data } \\
\hline Range (n. mi.) & 5,508 & 5,500 & 5,503 \\
\hline Landing $\mathrm{AOA}\left(^{\circ}\right)$ & 11.99 & 12.00 & 12.01 \\
\hline \multicolumn{4}{|c|}{ Optimized Weight for Design } \\
\hline$W_{g}(\mathrm{lbs})$ & 625,325 & 621,833 & 627,119 \\
\hline$W_{w}(\mathrm{lbs})$ & 83,382 & 84,456 & 82,915 \\
\hline$W_{b}(\mathrm{lbs})$ & 23,807 & 22,688 & 20,723 \\
\hline \multicolumn{4}{|c|}{ Other Wing Bending Material Weight Values } \\
\hline$W_{b_{\mathrm{G}}}(\mathrm{lbs})$ & 18,753 & 22,988 & 23,641 \\
\hline$W_{b_{\mathrm{F}}}(\mathrm{lbs})$ & 23,807 & 22,688 & 20,723 \\
\hline$W_{b_{\mathrm{RS} 1_{\mathrm{A}}}}(\mathrm{lbs})$ & 84,718 & 238,720 & 271,421 \\
\hline$W_{{b_{\mathrm{RS} 1}{ }_{\mathrm{AB}}}}(\mathrm{lbs})$ & 91,161 & 183,114 & 207,234 \\
\hline$W_{b_{\mathrm{RS} 2_{\mathrm{A}}}}(\mathrm{lbs})$ & 22,447 & 20,631 & 21,286 \\
\hline$W_{b_{\mathrm{RS} 2 \mathrm{AB}}}(\mathrm{lbs})$ & 23,007 & 21,318 & 21,931 \\
\hline$W_{b_{\mathrm{RS}{ }_{\mathrm{A}}}}(\mathrm{lbs})$ & 21,772 & 20,826 & 21,026 \\
\hline$W_{b_{\mathrm{RS} 3 \mathrm{AB}}}(\mathrm{lbs})$ & 21,378 & 20,396 & 20,604 \\
\hline
\end{tabular}
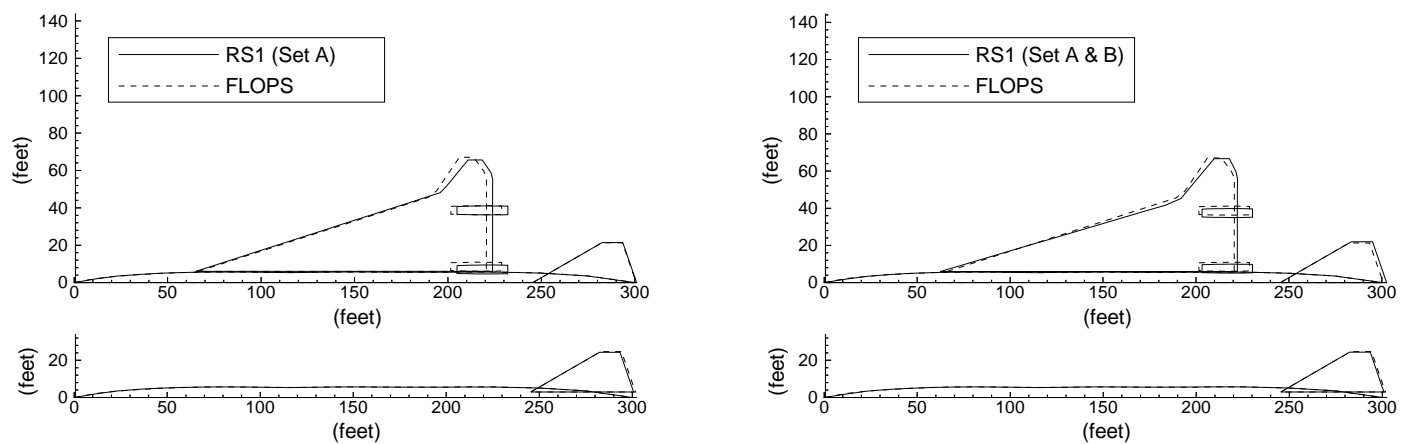

Figure 9.2: Optimal HSCT planforms using RS1. 
Table 9.4: Optimal HSCT designs using response surface RS2.

\begin{tabular}{|c|r|r|r|}
\hline Optimization $\rightarrow$ & FLOPS & RS2 ${ }_{\mathrm{A}}$ & RS2 ${ }_{\mathrm{AB}}$ \\
\hline \hline \multicolumn{4}{|c|}{ Planform Geometry } \\
\hline Root chord (ft) & 154.3 & 156.6 & 157.3 \\
\hline Tip chord (ft) & 7.53 & 7.17 & 7.54 \\
\hline Aspect Ratio & 1.77 & 1.75 & 1.74 \\
\hline Wing Area $\left(\mathrm{ft}^{2}\right)$ & 10,184 & 10,094 & 10,298 \\
\hline
\end{tabular}

Performance Data

\begin{tabular}{|c|r|r|r|}
\hline Range (n. mi.) & 5,508 & 5,500 & 5,503 \\
\hline Landing AOA $\left(^{\circ}\right)$ & 11.99 & 12.00 & 11.97 \\
\hline
\end{tabular}

\begin{tabular}{|c|r|r|r|}
\hline \multicolumn{4}{|c|}{ Optimized Weight for Design } \\
\hline$W_{g}(\mathrm{lbs})$ & 625,325 & 613,601 & 623,767 \\
\hline$W_{w}(\mathrm{lbs})$ & 83,382 & 77,222 & 80,125 \\
\hline$W_{b}(\mathrm{lbs})$ & 23,807 & 18,078 & 19,894 \\
\hline
\end{tabular}

Other Wing Bending Material Weight Values

\begin{tabular}{|c|r|r|r|}
\hline$W_{b_{\mathrm{G}}}(\mathrm{lbs})$ & 18,753 & 16,514 & 14,681 \\
\hline
\end{tabular}

\begin{tabular}{|l|r|r|r|}
\hline$W_{b_{\mathrm{F}}}(\mathrm{lbs})$ & 23,807 & 22,620 & 23,570 \\
\hline
\end{tabular}

\begin{tabular}{|c|r|r|r|}
\hline \hline$W_{b_{\mathrm{RS} 1_{\mathrm{A}}}}(\mathrm{lbs})$ & 84,718 & 154,591 & 169,384 \\
\hline$W_{b_{\mathrm{RS} 1_{\mathrm{AB}}}}(\mathrm{lbs})$ & 91,161 & 142,685 & 151,237 \\
\hline \hline$W_{b_{\mathrm{RS} 2}}(\mathrm{lbs})$ & 22,447 & 18,078 & 18,515 \\
\hline$W_{b_{\mathrm{RS} 2} \mathrm{AB}}(\mathrm{lbs})$ & 23,007 & 19,471 & 19,894 \\
\hline \hline$W_{b_{\mathrm{RS}{ }_{A}}}(\mathrm{lbs})$ & 21,772 & 20,770 & 21,573 \\
\hline$W_{b_{\mathrm{RS}{ }_{A} \mathrm{AB}}}(\mathrm{lbs})$ & 21,378 & 20,339 & 21,172 \\
\hline
\end{tabular}
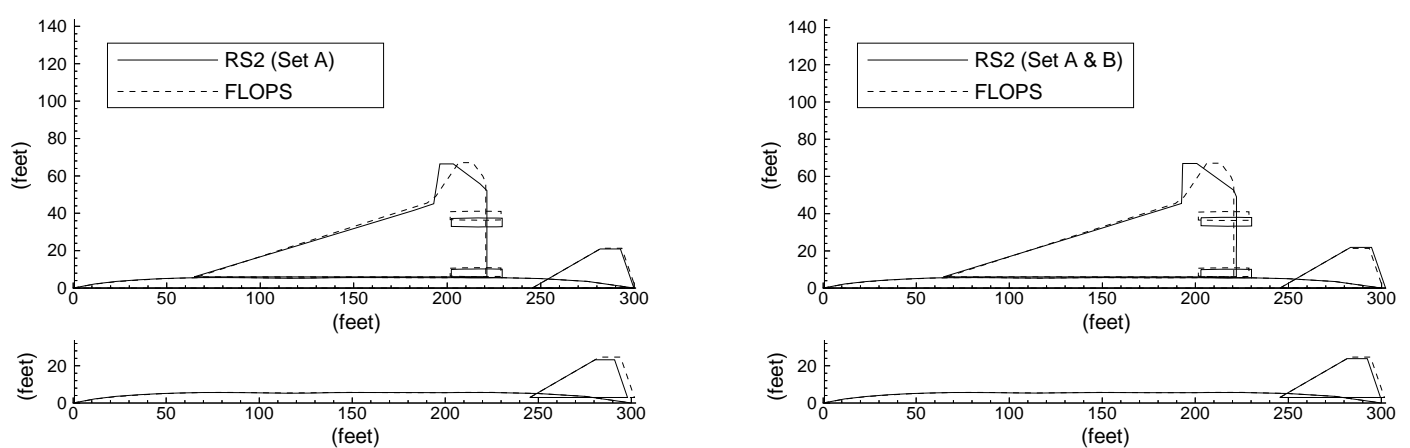

Figure 9.3: Optimal HSCT planforms using RS2. 
Table 9.5: Optimal HSCT designs using response surface RS3

\begin{tabular}{|c|c|c|c|}
\hline Optimization $\rightarrow$ & FLOPS & $\mathrm{RS} 3_{\mathrm{A}}$ & $\mathrm{RS} 3_{\mathrm{AB}}$ \\
\hline \multicolumn{4}{|c|}{ Planform Geometry } \\
\hline Root chord (ft) & 154.3 & 155.4 & 156.1 \\
\hline Tip chord (ft) & 7.53 & 7.71 & 7.60 \\
\hline Aspect Ratio & 1.77 & 1.74 & 1.79 \\
\hline Wing Area $\left(\mathrm{ft}^{2}\right)$ & 10,184 & 10,090 & 9,909 \\
\hline \multicolumn{4}{|c|}{ Performance Data } \\
\hline Range (n. mi.) & 5,508 & 5,507 & 5,503 \\
\hline Landing $\mathrm{AOA}\left({ }^{\circ}\right)$ & 11.99 & 12.02 & 12.06 \\
\hline \multicolumn{4}{|c|}{ Optimized Weight for Design } \\
\hline$W_{g}(\mathrm{lbs})$ & 625,325 & 617,309 & 619,245 \\
\hline$W_{w}(\mathrm{lbs})$ & 83,382 & 80,526 & 77,853 \\
\hline$W_{b}(\mathrm{lbs})$ & 23,807 & 21,706 & 20,083 \\
\hline \multicolumn{4}{|c|}{ Other Wing Bending Material Weight Values } \\
\hline$W_{b_{\mathrm{G}}}(\mathrm{lbs})$ & 18,753 & 29,083 & 22,164 \\
\hline$W_{b_{\mathrm{F}}}(\mathrm{lbs})$ & 23,807 & 23,731 & 22,335 \\
\hline$W_{b_{\mathrm{RS} 1}}(\mathrm{lbs})$ & 84,718 & 163,972 & 108,257 \\
\hline$W_{b_{\mathrm{RS} 1_{\mathrm{AB}}}}(\mathrm{lbs})$ & 91,161 & 165,081 & 113,321 \\
\hline$\overline{W_{b_{\mathrm{RS} 2} \mathrm{~A}}}(\mathrm{lbs})$ & 22,447 & 22,616 & 20,830 \\
\hline$W_{b_{\mathrm{RS} 2_{A \mathrm{~B}}}}(\mathrm{lbs})$ & 23,007 & 23,022 & 21,320 \\
\hline$W_{b_{\mathrm{RS} 3_{\mathrm{A}}}}(\mathrm{lbs})$ & 21,772 & 21,706 & 20,524 \\
\hline$W_{b_{\mathrm{RS} 3_{\mathrm{AB}}}}(\mathrm{lbs})$ & 21,378 & 21,309 & 20,083 \\
\hline
\end{tabular}
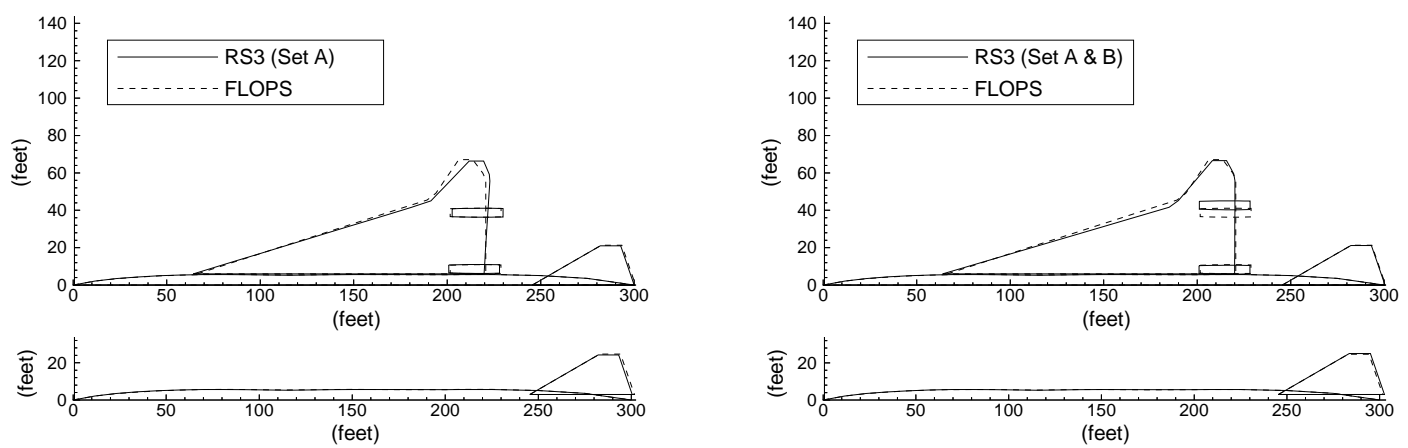

Figure 9.4: Optimal HSCT planforms using RS3. 


\section{Chapter 10}

\section{Concluding Remarks}

Variable-complexity response surface methods were developed for the calculation of wing bending material weight used in a HSCT configuration design. The response surface was based on the results of structural optimization. However, simple conceptualdesign level models were used to identify good variables for the response surface and to limit the extent of the design space. In particular, the variables used in algebraic weight equations in the FLOPS program were used to reduce the number of variables in the response surface from 25 to 10 . The use of the simple models to reduce the extent of the design space was shown to improve the accuracy of the response surface by several orders of magnitude.

Parallel computation permitted the execution of thousands of structural optimizations which were used for the creation of the response surface. Substantial noise in the results of the structural optimization, on the order of 20-30\%, was found and traced to noise in the loading data generated for each HSCT design. Six response surfaces were created with the structural optimization data using three different model functions. The most accurate response surface was characterized by an average error of about $10 \%$ when compared to structural optimization. Part of this error may attributed to the noise in structural optimization rather than to inaccuracies of the response surface.

Seven HSCT configuration optimizations were performed, one for each response surface, and one using the FLOPS weight equation. While the response surfaces did 
not produce significantly lighter HSCT designs, the bending material weight for the final designs was more accurate than that found using FLOPS.

Variable-complexity response surface approximations to the wing bending material weight for HSCT configuration optimization have been shown to be an effective way to deal with the expense of structural optimizations, the nonsmoothness of structural optima, and the practical difficulties of code integration. Practical considerations that balance response surface accuracy with its development cost are nontrivial. Achieving both acceptable accuracy and acceptable cost for response surface approximations to wing bending material weight remains an open question. The techniques described here are not limited to the wing bending material weight response and can be applied to other functions involved in HSCT design.

In future work, the sources of inaccuracy in the response surface approximations for wing bending material weight need to be positively identified and dealt with. In addition, alternatives to the $D$-optimal point will be considered. Other point selection methods may provide a means for minimizing errors associated with deficiencies in the model function, whereas the $D$-optimality approach assumes that noise in the data rather than the inadequacy of the response surface model is of paramount concern. 


\section{Bibliography}

[1] Unger, E. R., Hutchison, M. G., Rais-Rohani, M., Haftka, R. T., and Grossman, B., "Variable-Complexity Design of a Transport Wing," International Journal of Systems Automation: Research and Applications (SARA), no. 2, pp. 87-113, 1992.

[2] Hutchison, M. G., Unger, E. R., Mason, W. H., Grossman, B., and Haftka, R. T., "Variable-Complexity Aerodynamic Optimization of an HSCT Wing Using Structural Wing-Weight Equations," Journal of Aircraft, vol. 31, no. 1, pp. 110116, 1994.

[3] Dudley, J., Huang, X., MacMillin, P. E., Grossman, B., Haftka, R. T., and Mason, W. H., "Multidisciplinary Optimization of the High-Speed Civil Transport," AIAA 95-0124, Sept 1995. 33rd Aerospace Sciences Meeting and Exhibit. Reno, NV.

[4] Box, G. E. P. and Wilson, K. B., "On the Experimental Attainment of Optimum Conditions," Journal of Royal Statistical Society, vol. B13, pp. 38-45, 1951.

[5] Box, G. E. P., Hunter, W. G., and S., H. J., Statistics for Experiments: An Introduction to Design, Data Analysis, and Model Building. New York, N. Y.: John Wiley \& Sons, 1978.

[6] Myers, R. H. and Montgomery, D. C., Response Surface Methodology: Process and Product Optimization Using Designed Experiments, pp. 650-651. New York, N. Y.: John Wiley \& Sons, 1995. 
[7] Tai, J. C., Marvis, D. N., and Schrage, D. P., "Application of a Response Surface Method to the Design of Tipjet Driven Stopped Rotor/Wing Concepts," AIAA 95-3965, Sept 1995. 1st AIAA Aircraft Engineering, Technology, and Operations Congress. Los Angeles, CA.

[8] Engelund, W. C., Stanley, D. O., Lepsch, R. A., and McMillian, M. M., "Aerodynamic Configuration Design Using Response Methodology Analysis," AIAA 93-3967, Aug 1993. AIAA Aircraft Design, Systems, and Operations Meeting. Monterey, CA.

[9] Giunta, A. A., Balabanov, V., Burgee, S. L., Kaufman, M. D., Grossman, B., Mason, W. H., Haftka, R. T., and Watson, L. T., "Aerodynamic and Structural Optimization of a High Speed Civil Transport on Parallel Computers," May 1995. Proceedings of the First World Congress on Structural and Multidisciplinary Optimization. Goslar, Germany.

[10] Burgee, S. L., Giunta, A. A., Balabanov, V., Narducci, R., Grossman, B., Mason, W. H., Haftka, R. T., and Watson, L. T., "A Coarse Grained Parallel VariableComplexity Multidisciplinary Optimization Paradigm," Tech. Rep. TR 95-20, Virginia Polytechnic Institute and State University, Department of Computer Science, Oct 1995.

[11] Hutchison, M. G., Unger, E. R., Mason, W. H., Grossman, B., and Haftka, R. T., "Variable Complexity Aerodynamic-Structural Design of a High Speed Civil Transport Wing," AIAA 92-4695, Sept 1992. 4th AIAA/USAF/NASA/OAI Symposium on Multidisciplinary Analysis and Optimization. Cleveland, $\mathrm{OH}$.

[12] McCullers, L. A., "Aircraft Configuration Optimization Including Optimized Flight Profiles," in Proceedings of a Symposium on Recent Experiences in Multidisciplinary Analysis and Optimization (Sobieski, J., ed.), pp. 395-412, NASA Cp-2327, Apr 1984.

[13] York, P. and Labell, R., "Aircraft Weight Build-Up Methodology with modification for Materials and Construction Techniques," NASA CR-166173, 1980. 
[14] Huang, X., Haftka, R. T., Grossman, B., and Mason, W., "Comparison of Statistical-based Weight Equations with Structural Optimization for Supersonic Transport Wings," AIAA 94-4379, Sept 1994. 5th AIAA/USAF/NASA/ISSMO Symposium on Multidisciplinary Analysis and Optimization. Panama City, FL.

[15] Dudley, J., Huang, X., Haftka, R. T., Grossman, B., and Mason, W. H., "Variable-Complexity Interlacing of Weight Equation and Structural Optimization for the Design of the High Speed Civil Transport," AIAA 94-4377, Sept 1994. 5th AIAA/USAF/NASA/ISSMO Symposium on Multidisciplinary Analysis and Optimization. Panama City, FL.

[16] Malone, B. and Woyak, S. A., "An Object-Oriented Analysis and Optimization Control Environment for the Conceptual Design of Aircraft," AIAA 95-3862, Sept 1995.

[17] Woyak, S. A., Malone, B., and Myklebust, A., "An Architecture for Creating Engineering Applications: The Dynamic Integration System," in Proceedings of the Computers in Engineering Conference and the Engineering Database Symposium, (Boston, MA), pp. 1-8, ASME, Sept 1995.

[18] Box, M. J. and Draper, N. R., "Factorial Designs, the $\left|\mathbf{X}^{T} \mathbf{X}\right|$ Criterion, and Some Related Matters," Technometrics, vol. 13, no. 4, 1971.

[19] Vanderplaats, Miura and Associates, Inc, GENESIS User Manual, Version 1.3, Dec 1993.

[20] Giunta, A. A., Dudley, J. M., Narducci, R., Grossman, B., Haftka, R. T., Mason, W. H., and Watson, L. T., "Noisy Aerodynamic Response and Smooth Approximations in HSCT Design," AIAA 94-4376, Sept 1994. 5th AIAA/USAF/NASA/ISSMO Symposium on Multidisciplinary Analysis and Optimization. Panama City, FL.

[21] Mason, R. L., Gunst, R. F., and Hess, J. L., Statistical Design and Analysis of Experiments, pp. 215-221. New York, N. Y.: John Wiley \& Sons, 1989. 
[22] Hutchison, M. G., Unger, E. R., Mason, W. H., Grossman, B., and Haftka, R. T., "Aerodynamic Optimization of an HSCT Wing Using Variable-Complexity Modeling," AIAA 93-0101, Jan 1993. 31st Aerospace Sciences Meeting and Exhibit. Reno, NV.

[23] Hutchison, M. G., Multidisciplinary Optimization of High-Speed Civil Transport Configurations Using Variable Complexity Modeling. PhD thesis, Virginia Polytechnic and State University, Mar 1993.

[24] MacMillin, P., "Trim, Control, and Performance Effects in Variable-Complexity High-Speed Civil Transport Design," Master's thesis, Virginia Polytechnic and State University, Mar 1996.

[25] Grandhi, R. V., Thareja, R., and Haftka, R. T., "NEWSUMT-A: A General Purpose Program for Constrained Optimization Using Approximation," ASME Journal of Mechanisms, Transmissions and Automation in Design, vol. 107, pp. 94-99, 1985.

[26] Balabanov, V., Kaufman, M. K., Giunta, A. A., Haftka, R. T., Grossman, B., Mason, W. H., and Watson, L. T., "Developing Customized Wing Weight Function by Structural Optimization on Parallel Computers," AIAA 96-1336, April 1996. 37th AIAA/ASME/ASCE/AHS/ASC Structures, Structural Dynamics, and Materials Conference, Salt Lake City, UT.

[27] Huang, X., Structural Optimization and its Interaction with Aerodynamic Optimization for a High Speed Civil Transport. PhD thesis, Virginia Polytechnic and State University, Nov 1994.

[28] Barthelemy, J. F. M., Wrenn, G. A., R., D. A., Coen, P. G., and Hall, L. E., "Supersonic Transport Wing Minimum Weight Design Integrating Aerodynamics and Structures," Journal of Aircraft, vol. 31, Mar 1994.

[29] Carlson, H. W. and Walkley, K. B., "Numerical Methods and a Computer Program for Subsonic and Supersonic Aerodynamic Design and Analysis of Wings With Attainable Thrust Corrections," NASA CR 3808, 1984. 
[30] Hutchison, M. G., Huang, X., H., M. W., Haftka, R. T., and Grossman, B., "Variable-Complexity Aerodynamic-Structural Design of a High-Speed Civil Transport Wing," AIAA 92-4695, Sept 1992. 4th AIAA/USAF/NASA/OAI Symposium on Multidisciplinary Analysis and Optimization. Cleveland, OH.

[31] Khuri, A. I. and Cornell, J. A., Response Surfaces: Designs and Analyses. New York, N. Y.: Marcel Dekker, 1987.

[32] Box, G. E. P. and Behnken, D. W., "Some New Three Level Designs for the Study of Quantitative Variables," Technometrics, vol. 2, no. 4, pp. 455-475, 1960.

[33] Hinkelman, K., Design and analysis of experiments. New York, N. Y.: John Wiley \& Sons, Inc., 1994.

[34] SAS Institute Inc., JMP User's Guide Version 3.1, 1995.

[35] Narducci, R., Selected Optimization Procedures for CFD-based Shape Design Involving Shock Waves or Computational Noise. PhD thesis, Virginia Polytechnic and State University, 1995.

[36] Anderson, E., LAPACK User's Guide. Philadelphia: Society for Industrial and Applied Mathematics, 1992. 


\section{Appendix A}

\section{Least Squares Method and Analysis of Variance}

Statistical techniques known as regression analysis and analysis of variance (ANOVA) provide the means to identify and remove the less important terms in the response surface polynomial models based on simple analyses, thereby reducing the number of detailed analyses needed for later construction of accurate response surfaces. Regression analysis is the procedure by which the $c_{i}$ coefficients for the response surface model are obtained and typically involves the method of least squares, as follows. Let there be $m$ measurements $Y_{i}$ at $m$ distinct design points $\left(x_{1}^{(i)}, \ldots, x_{p}^{(i)}\right), i=1, \ldots$, $m$, and let the basis functions for the regression model be $\varphi_{1}(x), \ldots, \varphi_{n}(x)$, where $x=\left(x_{1}, \ldots, x_{p}\right)$. A choice for the $\varphi_{i}$ might be

$$
1, x_{1}, x_{2}, \ldots, x_{p}, x_{1}^{2}, x_{1} x_{2}, \ldots, x_{p}^{2}
$$

corresponding to a quadratic model. The regression model is

$$
y \approx c_{1} \varphi_{1}(x)+\cdots+c_{n} \varphi_{n}(x)
$$

which leads to the overdetermined matrix problem

$$
Y \approx \mathbf{X} c
$$


where $Y=\left(Y_{1}, \ldots, Y_{m}\right), c=\left(c_{1}, \ldots, c_{n}\right)$, and $\mathbf{X}$ is the $m \times n(m \geq n)$ matrix

$$
\left(\begin{array}{ccc}
\varphi_{1}\left(x^{(1)}\right) & \ldots & \varphi_{n}\left(x^{(1)}\right) \\
\vdots & \ddots & \vdots \\
\varphi_{1}\left(x^{(m)}\right) & \ldots & \varphi_{n}\left(x^{(m)}\right)
\end{array}\right) .
$$

The least squares solution is that (unique, assuming rank $\mathbf{X}=n$ ) vector $\tilde{c}$ which minimizes the 2-norm $\|Y-\mathbf{X} c\|_{2}$, or the sum of squares of the components of $Y-\mathbf{X} c$, the errors. The regression or response surface model of the data is then taken as

$$
\tilde{c}_{1} \varphi_{1}(x)+\cdots+\tilde{c}_{n} \varphi_{n}(x) .
$$

Matrix $\left(\mathbf{X}^{T} \mathbf{X}\right)^{-1}$ is called the variance-covariance matrix. The $n$ diagonal elements in this matrix are the variance values associated with the $n$ respective coefficients [31]. The standard deviation $\sigma_{k}$ of each coefficient is the square root of its variance.

Analysis of variance (ANOVA) involves estimating the variance of the predicted polynomial coefficients and uses the variance-covariance matrix $\left(\mathbf{X}^{T} \mathbf{X}\right)^{-1}$. The diagonal terms $\sigma_{i}{ }^{2}$ in this square matrix multiplied by the variance $\operatorname{Var}(\epsilon)$ in the measured function values $Y_{i}$ are the variances of the respective coefficients $c_{i}$ in the response surface polynomial model. The coefficient of variation $\Upsilon$ for each term in the polynomial is calculated as

$$
\Upsilon= \begin{cases}\frac{100\left|\sigma_{i}\right| \sqrt{\operatorname{Var}(\epsilon)}}{c_{i}}, & c_{i} \neq 0, \\ 100, & c_{i}=0,\end{cases}
$$

where the factor of 100 expresses the coefficient of variation as a percentage. The term $\sqrt{\operatorname{Var}(\epsilon)}$ is usually estimated by the RMS error of the least squares approximation at the $m$ data points. For the coefficient of variation calculations given below, $\operatorname{Var}(\epsilon)$ is taken to be unity. Terms in the polynomial model having large coefficients of variation, typically over 0.10 , may be dropped from the polynomial without significantly affecting the fidelity of the response surface fit. 


\section{Appendix B}

\section{FLOPS Weight Function Details}

The wing weight function in FLOPS is based on an analytic expression to relate wing bending material weight to wing geometry, material properties and loading. Two terms in the function account for the load distribution on the wing, the bending material factor, $B_{z}$, and the engine relief factor, $B_{z e}$.

$B_{z}$ accounts for the distribution of load on the wing, neglecting the engines, and is calculated by approximately determining the required material volume of the upper and lower skins in a simple wing box description of the wing. We begin by finding the weighted average of the load sweep angle at $75 \%$ chord position

$$
\Lambda_{L}=\int_{0}^{1}(1+2 y) \Lambda(y) d y \text {. }
$$

Next it is necessary to determine the bending moment assuming a simple elliptic pressure distribution.

$$
M(y)=\int_{0}^{y} p(\xi) \xi d \xi
$$

With this bending moment distribution it is possible to calculate the necessary flange are as

$$
A(y)=\frac{M(y)}{t(y) c(y) \cos \Lambda(y)},
$$

and the required volume as

$$
V=\int_{0}^{1} A(y) d y
$$

The total load is simply

$$
L=\int_{0}^{1} p(y) d y
$$


and the bending material factor is finally given as

$$
B_{z}=\frac{2 V}{L d}
$$

where

$$
\begin{gathered}
d=A R^{0.25}\left[1+\left(0.5 f_{a}-0.16\right) \sin ^{2} \Lambda_{L}+0.3 C_{y}\right. \\
\left.\left(1-0.5 f_{a}\right) \sin \Lambda_{L}\right] .
\end{gathered}
$$

$B_{z e}$ accounts for the reduced amount of structural weight necessary due to the presence of the engines on the wing.

$$
B_{z e}=16 \int_{y_{\text {root }}}^{y_{\text {tip }}} N E\left(\frac{y_{\text {tip }}-y}{\cos ^{2} \Lambda(y)}\right)\left(\frac{y_{\text {tip }}-y_{\text {root }}}{c(y) \operatorname{Max}(t / c)}\right) d y
$$

where $N E$ is given by

$$
N E= \begin{cases}2, & y<y_{\text {nacelle }_{1}} \\ 1, & y_{\text {nacelle }_{1}}<y<y_{\text {nacelle }_{2}} \\ 0, & y>y_{\text {nacell }_{2}}\end{cases}
$$




\section{Appendix C}

\section{Generation of Candidate Designs}

In the development of a response surface, a large number of data points that cover the design space are required. A systematic approach for producing the locations of these points has been developed assuming that the initial design space is a $p$-cube centered at the origin with vertices $( \pm 1, \ldots, \pm 1)$ and a quadratic $(2.1)$ is used for the model function. The level of a variable in a point selection system refers to the number of values that the variable is allowed to take on within a set of data points; for example, if a variable assumes the values $\{-1,0,+1\}$ within a set of points, then it is said to have a level of three [32].

The most general three-level point selection system is the full factorial method, where every variable assumes any of three possible values. For twenty-five variables, this requires the evaluation of $3^{25} \approx 8.5 \times 10^{11}$ designs, which is unrealistic. Consider instead a combination of two level-point selection systems based on the Partially Balanced Incomplete Box design (PBIB) [33] For a two-level system, each variable can take on the values $\{-1,+1\}$.

Similar to the PBIB design, a pattern of blocks is created, each of which contains a fraction of the total number of variables. The variables within a block are evaluated at the two levels \pm 1 , while the variables outside of the block are held fixed at the third level 0 . Three different blocking systems are incorporated to produce a satisfactory number of points. Every block pattern containing one, two, and three variables is considered, as well as the center point $(0, \ldots, 0)$. 
All possible two-variable block patterns for a problem in three variables, and the corresponding design points are given in Table C.1. For twenty-five variables, 19,651 points are produced using the three blocking systems and one center point. For an arbitrary number $p$ of variables the total number of points created is

$$
1+\sum_{i=1}^{3} 2^{i} \frac{p !}{i !(p-i) !} .
$$

Table C.1: Two-variable block pattern example.

\begin{tabular}{|c|c|c|c|}
\hline & $x_{1}$ & $x_{2}$ & $x_{3}$ \\
\hline \hline Block \#1 & $*$ & $*$ & \\
\hline Block \#2 & & $*$ & $*$ \\
\hline Block \#3 & $*$ & & $*$ \\
\hline
\end{tabular}

\begin{tabular}{|l|c|c|c|}
\hline & $x_{1}$ & $x_{2}$ & $x_{3}$ \\
\hline \hline Block \#1 & 1 & 1 & 0 \\
& -1 & 1 & 0 \\
& 1 & -1 & 0 \\
& -1 & -1 & 0 \\
\hline Block \#2 & 0 & 1 & 1 \\
& 0 & -1 & 1 \\
& 0 & 1 & -1 \\
& 0 & -1 & -1 \\
\hline Block \#3 & 1 & 0 & 1 \\
& -1 & 0 & 1 \\
& 1 & 0 & -1 \\
& -1 & 0 & -1 \\
\hline
\end{tabular}




\section{Appendix D}

\section{Response Surface Descriptions}

Details for several wing bending material weight response surfaces are presented in this appendix. While generating the response surfaces, numerical round-off error is minimized by scaling each variable $x_{i}$ to a value $x_{i}^{\prime}$ where

$$
\begin{aligned}
x_{i}^{\prime} & =a x_{i}-b, \\
a & =2 /\left(x_{i_{\max }}-x_{i_{\min }}\right), \\
b & =1+2 x_{i_{\min }} /\left(x_{i_{\max }}-x_{i_{\min }}\right),
\end{aligned}
$$

and $-1 \leq x_{i}^{\prime} \leq 1$. The terms $x_{i_{\min }}$ and $x_{i_{\max }}$ represent the maximum and minimum values for $x_{i}$ which occur in the data used to generate the response surface. The RSG program (Appendix F) is used to generate each response surface. Values for $a$ and $b$ are also generated automatically.

The $x_{i}$ variables for $\mathrm{RS} 1_{\mathrm{A}}$ and $\mathrm{RS} 1_{\mathrm{AB}}$ correspond in order to the twenty-five design variables listed in Table 3.1. For RS2 $2_{\mathrm{A}}$ and $\mathrm{RS} 2_{\mathrm{AB}}$ the $x_{i}$ correspond in order to the ten intervening variables listed in Table 4.1. $\mathrm{RS} 3_{\mathrm{A}}$ and $\mathrm{RS} 3_{\mathrm{AB}}$ have only one variable, the FLOPS prediction for wing bending material weight $W_{b_{\mathrm{F}}}$. 
Table D.1: Complete description of $\mathrm{RS}_{\mathrm{A}}$ (Part 1).

\begin{tabular}{|c|c|c||c|c|c|}
\hline Variable & $a$ & $b$ & Variable & $a$ & $b$ \\
\hline \hline$x_{1}$ & $3.320 \mathrm{e}+00$ & 5.367 & $x_{14}$ & $1.178 \mathrm{e}+01$ & 1.250 \\
\hline$x_{2}$ & $2.416 \mathrm{e}+00$ & 3.095 & $x_{15}$ & $2.049 \mathrm{e}+01$ & 3.500 \\
\hline$x_{3}$ & $5.650 \mathrm{e}-01$ & 2.547 & $x_{16}$ & $3.767 \mathrm{e}+00$ & 1.263 \\
\hline$x_{4}$ & $4.415 \mathrm{e}+00$ & 8.466 & $x_{17}$ & $9.437 \mathrm{e}-01$ & 1.250 \\
\hline$x_{5}$ & $2.540 \mathrm{e}-01$ & 1.326 & $x_{18}$ & $5.267 \mathrm{e}+00$ & 3.031 \\
\hline$x_{6}$ & $2.250 \mathrm{e}+00$ & 4.407 & $x_{19}$ & $9.137 \mathrm{e}-01$ & 1.606 \\
\hline$x_{7}$ & $3.570 \mathrm{e}+00$ & 3.500 & $x_{20}$ & $4.392 \mathrm{e}+00$ & 2.345 \\
\hline$x_{8}$ & $4.627 \mathrm{e}-01$ & 4.007 & $x_{21}$ & $8.472 \mathrm{e}-01$ & 1.597 \\
\hline$x_{9}$ & $4.057 \mathrm{e}-01$ & 1.326 & $x_{22}$ & $1.023 \mathrm{e}+00$ & 3.692 \\
\hline$x_{10}$ & $6.463 \mathrm{e}-01$ & 1.990 & $x_{23}$ & $1.632 \mathrm{e}+00$ & 5.581 \\
\hline$x_{11}$ & $8.396 \mathrm{e}-01$ & 2.263 & $x_{24}$ & $1.791 \mathrm{e}-01$ & 1.250 \\
\hline$x_{12}$ & $1.148 \mathrm{e}+00$ & 2.728 & $x_{25}$ & $1.753 \mathrm{e}-01$ & 1.250 \\
\hline$x_{13}$ & $1.024 \mathrm{e}+02$ & 3.049 & & & \\
\hline
\end{tabular}


Table D.2: Complete description of $\mathrm{RS}_{\mathrm{A}}$ (Part 2).

\begin{tabular}{|c|c|c|c|c|c|}
\hline Index & Basis Function & Coefficient & Index & Basis Function & Coefficient \\
\hline 1 & constant & $-1.142 \mathrm{e}+04$ & 32 & $x_{3}^{\prime} x_{17}^{\prime}$ & $4.565 \mathrm{e}+03$ \\
\hline 2 & $x_{1}^{\prime}$ & $-4.481 e+03$ & 33 & $x_{3}^{\prime} x_{22}^{\prime}$ & $9.904 \mathrm{e}+03$ \\
\hline 3 & $x_{2}^{\prime}$ & $9.033 \mathrm{e}+03$ & 34 & $x_{3}^{\prime} x_{25}^{\prime}$ & $5.822 \mathrm{e}+03$ \\
\hline 4 & $x_{3}^{\prime}$ & $-1.420 \mathrm{e}+04$ & 35 & $x_{4}^{\prime 2}$ & $-7.881 e+03$ \\
\hline 5 & $x_{5}^{\prime}$ & $1.194 \mathrm{e}+04$ & 36 & $x_{4}^{\prime} x_{6}^{\prime}$ & $1.322 \mathrm{e}+04$ \\
\hline 6 & $x_{6}^{\prime}$ & $-4.011 \mathrm{e}+04$ & 37 & $x_{4}^{\prime} x_{8}^{\prime}$ & $-8.361 e+03$ \\
\hline 7 & $x_{8}^{\prime}$ & $-7.005 \mathrm{e}+03$ & 38 & $x_{6}^{\prime 2}$ & $6.103 \mathrm{e}+03$ \\
\hline 8 & $x_{10}^{\prime}$ & $-3.105 \mathrm{e}+03$ & 39 & $x_{6}^{\prime} x_{7}^{\prime}$ & $-2.789 \mathrm{e}+03$ \\
\hline 9 & $x_{11}^{\prime}$ & $-5.645 \mathrm{e}+03$ & 40 & $x_{6}^{\prime} x_{8}^{\prime}$ & $1.033 \mathrm{e}+04$ \\
\hline 10 & $x_{13}^{\prime}$ & $-3.110 \mathrm{e}+04$ & 41 & $x_{6}^{\prime} x_{13}^{\prime}$ & $-5.365 \mathrm{e}+04$ \\
\hline 11 & $x_{15}^{\prime}$ & $-2.092 \mathrm{e}+03$ & 42 & $x_{6}^{\prime} x_{19}^{\prime}$ & $1.602 \mathrm{e}+04$ \\
\hline 12 & $x_{23}^{\prime}$ & $1.295 \mathrm{e}+04$ & 43 & $x_{8}^{\prime} x_{10}^{\prime}$ & $5.771 \mathrm{e}+03$ \\
\hline 13 & $x_{1}^{\prime 2}$ & $8.297 \mathrm{e}+03$ & 44 & $x_{8}^{\prime} x_{11}^{\prime}$ & $-3.613 e+03$ \\
\hline 14 & $x_{1}^{\prime} x_{2}^{\prime}$ & $-1.161 \mathrm{e}+04$ & 45 & $x_{8}^{\prime} x_{15}^{\prime}$ & $-4.377 \mathrm{e}+03$ \\
\hline 15 & $x_{1}^{\prime} x_{3}^{\prime}$ & $1.402 \mathrm{e}+04$ & 46 & $x_{8}^{\prime} x_{17}^{\prime}$ & $5.764 \mathrm{e}+03$ \\
\hline 16 & $x_{1}^{\prime} x_{5}^{\prime}$ & $-1.960 \mathrm{e}+04$ & 47 & $x_{8}^{\prime} x_{18}^{\prime}$ & $-1.100 \mathrm{e}+04$ \\
\hline 17 & $x_{1}^{\prime} x_{6}^{\prime}$ & $4.194 \mathrm{e}+03$ & 48 & $x_{8}^{\prime} x_{20}^{\prime}$ & $-5.761 e+03$ \\
\hline 18 & $x_{1}^{\prime} x_{20}^{\prime}$ & $-4.988 \mathrm{e}+03$ & 49 & $x_{8}^{\prime} x_{21}^{\prime}$ & $2.144 \mathrm{e}+03$ \\
\hline 19 & $x_{1}^{\prime} x_{24}^{\prime}$ & $-4.962 \mathrm{e}+03$ & 50 & $x_{8}^{\prime} x_{24}^{\prime}$ & $3.352 \mathrm{e}+03$ \\
\hline 20 & $x_{2}^{\prime 2}$ & $-1.122 \mathrm{e}+04$ & 51 & $x_{8}^{\prime} x_{25}^{\prime}$ & $2.591 \mathrm{e}+03$ \\
\hline 21 & $x_{2}^{\prime} x_{4}^{\prime}$ & $5.279 \mathrm{e}+03$ & 52 & $x_{9}^{\prime} x_{18}^{\prime}$ & $3.495 \mathrm{e}+03$ \\
\hline 22 & $x_{2}^{\prime} x_{6}^{\prime}$ & $-1.693 e+04$ & 53 & $x_{10}^{\prime 2}$ & $2.993 \mathrm{e}+03$ \\
\hline 23 & $x_{2}^{\prime} x_{8}^{\prime}$ & $1.769 \mathrm{e}+04$ & 54 & $x_{11}^{\prime}{ }^{2}$ & $1.239 \mathrm{e}+03$ \\
\hline 24 & $x_{2}^{\prime} x_{19}^{\prime}$ & $1.349 \mathrm{e}+04$ & 55 & $x_{17}^{\prime} x_{19}^{\prime}$ & $2.763 \mathrm{e}+03$ \\
\hline 25 & $x_{2}^{\prime} x_{23}^{\prime}$ & $-1.558 \mathrm{e}+04$ & 56 & $x_{18}^{\prime 2}$ & $9.768 \mathrm{e}+02$ \\
\hline 26 & $x_{3}^{\prime} x_{4}^{\prime}$ & $-6.351 e+03$ & 57 & $x_{18}^{\prime} x_{19}^{\prime}$ & $-5.655 \mathrm{e}+03$ \\
\hline 27 & $x_{3}^{\prime} x_{5}^{\prime}$ & $4.769 \mathrm{e}+03$ & 58 & $x_{18}^{\prime} x_{22}^{\prime}$ & $5.940 \mathrm{e}+03$ \\
\hline 28 & $x_{3}^{\prime} x_{6}^{\prime}$ & $1.409 \mathrm{e}+04$ & 59 & $x_{19}^{\prime 2}$ & $-2.756 \mathrm{e}+03$ \\
\hline 29 & $x_{3}^{\prime} x_{7}^{\prime}$ & $-4.051 \mathrm{e}+03$ & 60 & $x_{21}^{\prime} x_{22}^{\prime}$ & $-2.675 \mathrm{e}+03$ \\
\hline 30 & $x_{3}^{\prime} x_{8}^{\prime}$ & $-7.280 \mathrm{e}+03$ & 61 & $x_{22}^{\prime} x_{24}^{\prime}$ & $-1.275 \mathrm{e}+04$ \\
\hline 31 & $x_{3}^{\prime} x_{11}^{\prime}$ & $6.127 \mathrm{e}+03$ & & & \\
\hline
\end{tabular}


Table D.3: Complete description of $\mathrm{RS}_{\mathrm{AB}}$ (Part 1).

\begin{tabular}{|c|c|c||c|c|c|}
\hline Variable & $a$ & $b$ & Variable & $a$ & $b$ \\
\hline \hline$x_{1}$ & $3.320 \mathrm{e}+00$ & 5.367 & $x_{14}$ & $1.178 \mathrm{e}+01$ & 1.250 \\
\hline$x_{2}$ & $1.932 \mathrm{e}+00$ & 2.675 & $x_{15}$ & $2.049 \mathrm{e}+01$ & 3.500 \\
\hline$x_{3}$ & $5.650 \mathrm{e}-01$ & 2.547 & $x_{16}$ & $3.767 \mathrm{e}+00$ & 1.263 \\
\hline$x_{4}$ & $3.506 \mathrm{e}+00$ & 6.770 & $x_{17}$ & $9.437 \mathrm{e}-01$ & 1.250 \\
\hline$x_{5}$ & $2.540 \mathrm{e}-01$ & 1.326 & $x_{18}$ & $5.267 \mathrm{e}+00$ & 3.031 \\
\hline$x_{6}$ & $2.240 \mathrm{e}+00$ & 4.383 & $x_{19}$ & $8.359 \mathrm{e}-01$ & 1.416 \\
\hline$x_{7}$ & $3.570 \mathrm{e}+00$ & 3.500 & $x_{20}$ & $4.392 \mathrm{e}+00$ & 2.345 \\
\hline$x_{8}$ & $4.627 \mathrm{e}-01$ & 4.007 & $x_{21}$ & $8.472 \mathrm{e}-01$ & 1.597 \\
\hline$x_{9}$ & $3.990 \mathrm{e}-01$ & 1.321 & $x_{22}$ & $1.023 \mathrm{e}+00$ & 3.692 \\
\hline$x_{10}$ & $6.463 \mathrm{e}-01$ & 1.990 & $x_{23}$ & $1.632 \mathrm{e}+00$ & 5.581 \\
\hline$x_{11}$ & $8.396 \mathrm{e}-01$ & 2.263 & $x_{24}$ & $1.791 \mathrm{e}-01$ & 1.250 \\
\hline$x_{12}$ & $1.148 \mathrm{e}+00$ & 2.728 & $x_{25}$ & $1.753 \mathrm{e}-01$ & 1.250 \\
\hline$x_{13}$ & $1.024 \mathrm{e}+02$ & 3.049 & & & \\
\hline
\end{tabular}


Table D.4: Complete description of $\mathrm{RS}_{\mathrm{AB}}$ (Part 2).

\begin{tabular}{|c|c|c|c|c|c|}
\hline Index & Basis Function & Coefficient & Index & Basis Function & Coefficient \\
\hline 1 & constant & $-2.343 \mathrm{e}+03$ & 32 & $x_{3}^{\prime} x_{17}^{\prime}$ & $4.067 \mathrm{e}+03$ \\
\hline 2 & $x_{1}^{\prime}$ & $-6.584 e+03$ & 33 & $x_{3}^{\prime} x_{22}^{\prime}$ & $7.064 \mathrm{e}+03$ \\
\hline 3 & $x_{2}^{\prime}$ & $1.737 \mathrm{e}+04$ & 34 & $x_{3}^{\prime} x_{25}^{\prime}$ & $4.902 \mathrm{e}+03$ \\
\hline 4 & $x_{3}^{\prime}$ & $-1.097 \mathrm{e}+04$ & 35 & $x_{4}^{\prime 2}$ & $-1.010 \mathrm{e}+04$ \\
\hline 5 & $x_{5}^{\prime}$ & $1.155 \mathrm{e}+04$ & 36 & $x_{4}^{\prime} x_{6}^{\prime}$ & $1.646 \mathrm{e}+04$ \\
\hline 6 & $x_{6}^{\prime}$ & $-2.462 \mathrm{e}+04$ & 37 & $x_{4}^{\prime} x_{8}^{\prime}$ & $-5.579 \mathrm{e}+03$ \\
\hline 7 & $x_{8}^{\prime}$ & $-1.158 \mathrm{e}+03$ & 38 & $x_{6}^{\prime 2}$ & $5.885 \mathrm{e}+03$ \\
\hline 8 & $x_{10}^{\prime}$ & $-3.709 \mathrm{e}+03$ & 39 & $x_{6}^{\prime} x_{7}^{\prime}$ & $-3.023 \mathrm{e}+03$ \\
\hline 9 & $x_{11}^{\prime}$ & $-5.987 \mathrm{e}+03$ & 40 & $x_{6}^{\prime} x_{8}^{\prime}$ & $8.835 \mathrm{e}+03$ \\
\hline 10 & $x_{13}^{\prime}$ & $-1.936 \mathrm{e}+04$ & 41 & $x_{6}^{\prime} x_{13}^{\prime}$ & $-3.358 \mathrm{e}+04$ \\
\hline 11 & $x_{15}^{\prime}$ & $-1.378 \mathrm{e}+03$ & 42 & $x_{6}^{\prime} x_{19}^{\prime}$ & $6.851 \mathrm{e}+03$ \\
\hline 12 & $x_{23}^{\prime}$ & $4.151 \mathrm{e}+03$ & 43 & $x_{8}^{\prime} x_{10}^{\prime}$ & $4.376 \mathrm{e}+03$ \\
\hline 13 & $x_{1}^{\prime 2}$ & $8.415 \mathrm{e}+03$ & 44 & $x_{8}^{\prime} x_{11}^{\prime}$ & $-4.168 \mathrm{e}+03$ \\
\hline 14 & $x_{1}^{\prime} x_{2}^{\prime}$ & $-1.638 \mathrm{e}+04$ & 45 & $x_{8}^{\prime} x_{15}^{\prime}$ & $-3.188 \mathrm{e}+03$ \\
\hline 15 & $x_{1}^{\prime} x_{3}^{\prime}$ & $1.434 \mathrm{e}+04$ & 46 & $x_{8}^{\prime} x_{17}^{\prime}$ & $3.922 \mathrm{e}+03$ \\
\hline 16 & $x_{1}^{\prime} x_{5}^{\prime}$ & $-1.846 \mathrm{e}+04$ & 47 & $x_{8}^{\prime} x_{18}^{\prime}$ & $-7.214 \mathrm{e}+03$ \\
\hline 17 & $x_{1}^{\prime} x_{6}^{\prime}$ & $4.446 \mathrm{e}+03$ & 48 & $x_{8}^{\prime} x_{20}^{\prime}$ & $-3.153 \mathrm{e}+03$ \\
\hline 18 & $x_{1}^{\prime} x_{20}^{\prime}$ & $-2.768 \mathrm{e}+03$ & 49 & $x_{8}^{\prime} x_{21}^{\prime}$ & $2.258 \mathrm{e}+03$ \\
\hline 19 & $x_{1}^{\prime} x_{24}^{\prime}$ & $-2.343 \mathrm{e}+03$ & 50 & $x_{8}^{\prime} x_{24}^{\prime}$ & $2.895 \mathrm{e}+03$ \\
\hline 20 & $x_{2}^{\prime 2}$ & $-7.618 e+03$ & 51 & $x_{8}^{\prime} x_{25}^{\prime}$ & $2.053 \mathrm{e}+03$ \\
\hline 21 & $x_{2}^{\prime} x_{4}^{\prime}$ & $1.724 \mathrm{e}+04$ & 52 & $x_{9}^{\prime} x_{18}^{\prime}$ & $3.205 \mathrm{e}+03$ \\
\hline 22 & $x_{2}^{\prime} x_{6}^{\prime}$ & $-1.645 e+04$ & 53 & $x_{10}^{\prime 2}$ & $3.515 \mathrm{e}+03$ \\
\hline 23 & $x_{2}^{\prime} x_{8}^{\prime}$ & $2.098 \mathrm{e}+04$ & 54 & $x_{11}^{\prime 2}$ & $1.327 \mathrm{e}+03$ \\
\hline 24 & $x_{2}^{\prime} x_{19}^{\prime}$ & $9.622 \mathrm{e}+03$ & 55 & $x_{17}^{\prime} x_{19}^{\prime}$ & $1.466 \mathrm{e}+03$ \\
\hline 25 & $x_{2}^{\prime} x_{23}^{\prime}$ & $-5.020 e+03$ & 56 & $x_{18}^{\prime}{ }^{2}$ & $9.291 \mathrm{e}+02$ \\
\hline 26 & $x_{3}^{\prime} x_{4}^{\prime}$ & $-7.355 \mathrm{e}+03$ & 57 & $x_{18}^{\prime} x_{19}^{\prime}$ & $-4.347 \mathrm{e}+03$ \\
\hline 27 & $x_{3}^{\prime} x_{5}^{\prime}$ & $3.427 \mathrm{e}+03$ & 58 & $x_{18}^{\prime} x_{22}^{\prime}$ & $2.796 \mathrm{e}+03$ \\
\hline 28 & $x_{3}^{\prime} x_{6}^{\prime}$ & $1.644 \mathrm{e}+04$ & 59 & $x_{19}^{\prime 2}$ & $-1.574 \mathrm{e}+03$ \\
\hline 29 & $x_{3}^{\prime} x_{7}^{\prime}$ & $-4.009 e+03$ & 60 & $x_{21}^{\prime} x_{22}^{\prime}$ & $-2.297 \mathrm{e}+03$ \\
\hline 30 & $x_{3}^{\prime} x_{8}^{\prime}$ & $-1.165 e+03$ & 61 & $x_{22}^{\prime} x_{24}^{\prime}$ & $-7.727 \mathrm{e}+03$ \\
\hline 31 & $x_{3}^{\prime} x_{11}^{\prime}$ & $5.965 \mathrm{e}+03$ & & & \\
\hline
\end{tabular}


Table D.5: Complete description of $\mathrm{RS} 2_{\mathrm{A}}$.

\begin{tabular}{|c|c|c||c|c|c|}
\hline Variable & $a$ & $b$ & Variable & $a$ & $b$ \\
\hline \hline$x_{1}$ & $8.765 \mathrm{e}-04$ & 1.250 & $x_{6}$ & $4.348 \mathrm{e}-02$ & 1.493 \\
\hline$x_{2}$ & $1.791 \mathrm{e}-03$ & 1.250 & $x_{7}$ & $1.269 \mathrm{e}-01$ & 1.652 \\
\hline$x_{3}$ & $4.577 \mathrm{e}-01$ & 5.950 & $x_{8}$ & $4.049 \mathrm{e}-04$ & 5.073 \\
\hline$x_{4}$ & $2.313 \mathrm{e}-02$ & 4.285 & $x_{9}$ & $1.632 \mathrm{e}-05$ & 5.581 \\
\hline$x_{5}$ & $3.336 \mathrm{e}+00$ & 3.005 & $x_{10}$ & $1.360 \mathrm{e}-05$ & 9.194 \\
\hline
\end{tabular}

\begin{tabular}{|c|c|c||c|c|c|}
\hline Index & Basis Function & Coefficient & Index & Basis Function & Coefficient \\
\hline \hline 1 & constant & $3.959 \mathrm{e}+04$ & 9 & $x_{8}^{\prime}$ & $4.448 \mathrm{e}+03$ \\
\hline 2 & $x_{1}^{\prime}$ & $4.558 \mathrm{e}+03$ & 10 & $x_{9}^{\prime}$ & $4.383 \mathrm{e}+04$ \\
\hline 3 & $x_{2}^{\prime}$ & $1.110 \mathrm{e}+03$ & 11 & $x_{10}^{\prime}$ & $-4.446 \mathrm{e}+04$ \\
\hline 4 & $x_{3}^{\prime}$ & $9.522 \mathrm{e}+03$ & 12 & $x_{4}^{\prime} x_{7}^{\prime}$ & $8.675 \mathrm{e}+03$ \\
\hline 5 & $x_{4}^{\prime}$ & $1.954 \mathrm{e}+04$ & 13 & $x_{5}^{\prime 2}$ & $-3.496 \mathrm{e}+03$ \\
\hline 6 & $x_{5}^{\prime}$ & $2.728 \mathrm{e}+03$ & 14 & $x_{7}^{\prime 2}$ & $-1.124 \mathrm{e}+04$ \\
\hline 7 & $x_{6}^{\prime}$ & $-1.857 \mathrm{e}+03$ & 15 & $x_{7}^{\prime} x_{8}^{\prime}$ & $-7.575 \mathrm{e}+03$ \\
\hline 8 & $x_{7}^{\prime}$ & $2.810 \mathrm{e}+04$ & & & \\
\hline
\end{tabular}

Table D.6: Complete description of RS2 $2_{\mathrm{AB}}$.

\begin{tabular}{|c|c|c||c|c|c|}
\hline Variable & $a$ & $b$ & Variable & $a$ & $b$ \\
\hline \hline$x_{1}$ & $8.765 \mathrm{e}-04$ & 1.250 & $x_{6}$ & $3.971 \mathrm{e}-02$ & 1.450 \\
\hline$x_{2}$ & $1.791 \mathrm{e}-03$ & 1.250 & $x_{7}$ & $1.269 \mathrm{e}-01$ & 1.652 \\
\hline$x_{3}$ & $4.435 \mathrm{e}-01$ & 5.735 & $x_{8}$ & $4.049 \mathrm{e}-04$ & 5.073 \\
\hline$x_{4}$ & $2.313 \mathrm{e}-02$ & 4.285 & $x_{9}$ & $1.632 \mathrm{e}-05$ & 5.581 \\
\hline$x_{5}$ & $3.336 \mathrm{e}+00$ & 3.005 & $x_{10}$ & $1.314 \mathrm{e}-05$ & 8.913 \\
\hline
\end{tabular}

\begin{tabular}{|c|c|c||c|c|c|}
\hline Index & Basis Function & Coefficient & Index & Basis Function & Coefficient \\
\hline \hline 1 & constant & $4.074 \mathrm{e}+04$ & 9 & $x_{8}^{\prime}$ & $7.954 \mathrm{e}+03$ \\
\hline 2 & $x_{1}^{\prime}$ & $5.947 \mathrm{e}+03$ & 10 & $x_{9}^{\prime}$ & $5.626 \mathrm{e}+04$ \\
\hline 3 & $x_{2}^{\prime}$ & $1.597 \mathrm{e}+03$ & 11 & $x_{10}^{\prime}$ & $-6.012 \mathrm{e}+04$ \\
\hline 4 & $x_{3}^{\prime}$ & $1.256 \mathrm{e}+04$ & 12 & $x_{4}^{\prime} x_{7}^{\prime}$ & $1.295 \mathrm{e}+04$ \\
\hline 5 & $x_{4}^{\prime}$ & $2.206 \mathrm{e}+04$ & 13 & $x_{5}^{\prime 2}$ & $-3.975 \mathrm{e}+03$ \\
\hline 6 & $x_{5}^{\prime}$ & $2.324 \mathrm{e}+03$ & 14 & $x_{7}^{\prime 2}$ & $-1.113 \mathrm{e}+04$ \\
\hline 7 & $x_{6}^{\prime}$ & $-2.471 \mathrm{e}+03$ & 15 & $x_{7}^{\prime} x_{8}^{\prime}$ & $-7.731 \mathrm{e}+03$ \\
\hline 8 & $x_{7}^{\prime}$ & $3.634 \mathrm{e}+04$ & & & \\
\hline
\end{tabular}


Table D.7: Complete description of $\mathrm{RS} 3_{\mathrm{A}}$.

\begin{tabular}{|c|c|c||c|c|c|}
\hline Variable & $a$ & $b$ & Variable & $a$ & $b$ \\
\hline \hline$x_{1}$ & $3.796 \mathrm{e}-05$ & 1.759 & & & \\
\hline
\end{tabular}

\begin{tabular}{|c|c|c||c|c|c|}
\hline Index & Basis Function & Coefficient & Index & Basis Function & Coefficient \\
\hline \hline 1 & constant & $-3.649 \mathrm{e}-01$ & 2 & $x_{1}^{\prime}$ & $5.380 \mathrm{e}-01$ \\
\hline
\end{tabular}

Table D.8: Complete description of RS3 $3_{\mathrm{AB}}$.

\begin{tabular}{|c|c|c||c|c|c|}
\hline Variable & $a$ & $b$ & Variable & $a$ & $b$ \\
\hline \hline$x_{1}$ & $3.796 \mathrm{e}-05$ & 1.759 & & & \\
\hline
\end{tabular}

\begin{tabular}{|c|c|c||c|c|c|}
\hline Index & Basis Function & Coefficient & Index & Basis Function & Coefficient \\
\hline \hline 1 & constant & $-3.596 \mathrm{e}-01$ & 2 & $x_{1}^{\prime}$ & $5.522 \mathrm{e}-01$ \\
\hline
\end{tabular}




\section{Appendix E}

\section{Optimal HSCT Designs}

As described in Chapter 3, each HSCT configuration is described by twenty-eight design variables. Design variable values for each of the seven HSCT design optimizations performed in Chapter 8 are presented in this appendix. The baseline design given Table E.1 was used as the starting point for each optimization and is identical to the baseline design described in Chapter 3 . 
Table E.1: Design variables for baseline HSCT and optimal design using FLOPS.

\begin{tabular}{|c|c|c|c|c|}
\hline$\#$ & Baseline & FLOPS & Multiplier & Description \\
\hline$\overline{1}$ & 1.814780 & 1.543042 & 100 & Wing root chord (ft) \\
\hline 2 & 1.559070 & 1.258446 & 100 & LE Break, $x(\mathrm{ft})$ \\
\hline 3 & 4.923480 & 4.045171 & 10 & LE Break, $y(\mathrm{ft})$ \\
\hline 4 & 1.816030 & 1.543603 & 100 & TE Break, $x(\mathrm{ft})$ \\
\hline 5 & 6.424340 & 5.177650 & 10 & TE Break, $y(\mathrm{ft})$ \\
\hline 6 & 1.695730 & 1.398542 & 100 & LE of wing tip, $x(\mathrm{ft})$ \\
\hline 7 & 0.700360 & 0.753067 & 10 & Tip chord (ft) \\
\hline 8 & 7.590870 & 6.107071 & 10 & Wing semi-span (ft) \\
\hline 9 & 4.019410 & 4.006502 & 0.10 & Chordwise location of max. $t / c$ \\
\hline 10 & 3.692140 & 2.748246 & 1 & Airfoil LE radius parameter, $r_{t}$ \\
\hline 11 & 2.578860 & 2.571982 & 0.01 & Airfoil $t / c$ at root \\
\hline 12 & 2.159350 & 1.610661 & 0.01 & Airfoil $t / c$ at LE break \\
\hline 13 & 1.803900 & 1.536393 & 0.01 & Airfoil $t / c$ at tip \\
\hline 14 & 0.021960 & 0.023630 & 100 & Fuselage restraint $1, x(\mathrm{ft})$ \\
\hline 15 & 0.106100 & 0.049342 & 10 & Fuselage restraint $1, r(\mathrm{ft})$ \\
\hline 16 & 0.122000 & 0.125346 & 100 & Fuselage restraint $2, x(\mathrm{ft})$ \\
\hline 17 & 0.349610 & 0.227605 & 10 & Fuselage restraint $2, r(\mathrm{ft})$ \\
\hline 18 & 1.324630 & 1.146570 & 100 & Fuselage restraint $3, x(\mathrm{ft})$ \\
\hline 19 & 0.534100 & 0.537699 & 10 & Fuselage restraint $3, r(\mathrm{ft})$ \\
\hline 20 & 2.486680 & 1.862943 & 100 & Fuselage restraint $4, x(\mathrm{ft})$ \\
\hline 21 & 0.466610 & 0.544783 & 10 & Fuselage restraint $4, r(\mathrm{ft})$ \\
\hline 22 & 2.623010 & 0.851736 & 10 & Nacelle $1, x_{9}(\mathrm{ft})$ \\
\hline 23 & 3.238760 & 3.871616 & 10 & Nacelle $2, x_{10}(\mathrm{ft})$ \\
\hline 24 & 3.226170 & 3.294296 & 100000 & Mission fuel (lbs) \\
\hline 25 & 6.479430 & 5.785234 & 10000 & Starting cruise altitude (ft) \\
\hline 26 & 0.338980 & 0.377398 & 100 & Cruise climb rate $(\mathrm{ft} / \mathrm{min})$ \\
\hline 27 & 6.978540 & 7.311565 & 100 & Vertical tail area $\left(\mathrm{ft}^{2}\right)$ \\
\hline 28 & 7.130590 & 7.063809 & 100 & Horizontal tail area $\left(\mathrm{ft}^{2}\right)$ \\
\hline
\end{tabular}


Table E.2: Design variables for optimal HSCT design using RS1.

\begin{tabular}{|c|c|c|c|c|}
\hline$\#$ & $\overline{\mathrm{RS}} 1_{\mathrm{A}}$ & $\overline{\mathrm{RS}} 1_{\mathrm{AB}}$ & Multiplier & Description \\
\hline 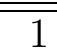 & 1.588858 & 1.597588 & 100 & Wing root chord (ft) \\
\hline 2 & 1.315947 & 1.281843 & 100 & LE Break, $x(\mathrm{ft})$ \\
\hline 3 & 4.231596 & 3.776931 & 10 & LE Break, $y(\mathrm{ft})$ \\
\hline 4 & 1.589588 & 1.597778 & 100 & TE Break, $x(\mathrm{ft})$ \\
\hline 5 & 5.151052 & 5.213062 & 10 & TE Break, $y(\mathrm{ft})$ \\
\hline 6 & 1.457859 & 1.472290 & 100 & LE of wing tip, $x(\mathrm{ft})$ \\
\hline 7 & 0.758825 & 0.821930 & 10 & Tip chord (ft) \\
\hline 8 & 5.962635 & 6.065901 & 10 & Wing semi-span (ft) \\
\hline 9 & 3.984763 & 3.868125 & 0.10 & Chordwise location of max. $t / c$ \\
\hline 10 & 3.097659 & 2.818636 & 1 & Airfoil LE radius parameter, $r_{t}$ \\
\hline 11 & 2.433507 & 2.700334 & 0.01 & Airfoil $t / c$ at root \\
\hline 12 & 1.634961 & 1.845030 & 0.01 & Airfoil $t / c$ at LE break \\
\hline 13 & 1.640434 & 1.532088 & 0.01 & Airfoil $t / c$ at tip \\
\hline 14 & 0.050612 & 0.057530 & 100 & Fuselage restraint $1, x(\mathrm{ft})$ \\
\hline 15 & 0.036121 & 0.029286 & 10 & Fuselage restraint $1, r(\mathrm{ft})$ \\
\hline 16 & 0.151024 & 0.157590 & 100 & Fuselage restraint $2, x(\mathrm{ft})$ \\
\hline 17 & 0.259453 & 0.257664 & 10 & Fuselage restraint $2, r(\mathrm{ft})$ \\
\hline 18 & 1.141982 & 1.142866 & 100 & Fuselage restraint $3, x(\mathrm{ft})$ \\
\hline 19 & 0.533511 & 0.535911 & 10 & Fuselage restraint $3, r(\mathrm{ft})$ \\
\hline 20 & 1.871758 & 1.866251 & 100 & Fuselage restraint $4, x(\mathrm{ft})$ \\
\hline 21 & 0.546410 & 0.546388 & 10 & Fuselage restraint $4, r(\mathrm{ft})$ \\
\hline 22 & 0.700839 & 0.751011 & 10 & Nacelle $1, x_{9}(\mathrm{ft})$ \\
\hline 23 & 3.863262 & 3.741006 & 10 & Nacelle $2, x_{10}(\mathrm{ft})$ \\
\hline 24 & 3.247413 & 3.311607 & 100000 & Mission fuel (lbs) \\
\hline 25 & 5.820271 & 5.758425 & 10000 & Starting cruise altitude (ft) \\
\hline 26 & 0.369611 & 0.385835 & 100 & Cruise climb rate $(\mathrm{ft} / \mathrm{min})$ \\
\hline 27 & 7.066911 & 7.093021 & 100 & Vertical tail area $\left(\mathrm{ft}^{2}\right)$ \\
\hline 28 & 7.124586 & 7.535361 & 100 & Horizontal tail area $\left(\mathrm{ft}^{2}\right)$ \\
\hline
\end{tabular}


Table E.3: Design variables for optimal HSCT design using RS2.

\begin{tabular}{|c|c|c|c|c|}
\hline$\#$ & $\overline{\mathrm{RS} 2} 2_{\mathrm{A}}$ & $\mathrm{RS} 2_{\mathrm{AB}}$ & Multiplier & Description \\
\hline 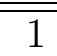 & 1.566197 & 1.573494 & 100 & Wing root chord (ft) \\
\hline 2 & 1.281543 & 1.279532 & 100 & LE Break, $x(\mathrm{ft})$ \\
\hline 3 & 3.876348 & 3.944267 & 10 & LE Break, $y(\mathrm{ft})$ \\
\hline 4 & 1.566305 & 1.574227 & 100 & TE Break, $x(\mathrm{ft})$ \\
\hline 5 & 4.704028 & 4.554112 & 10 & TE Break, $y(\mathrm{ft})$ \\
\hline 6 & 1.314562 & 1.285337 & 100 & LE of wing tip, $x(\mathrm{ft})$ \\
\hline 7 & 0.716578 & 0.753555 & 10 & Tip chord (ft) \\
\hline 8 & 6.040580 & 6.098442 & 10 & Wing semi-span (ft) \\
\hline 9 & 3.943968 & 3.970176 & 0.10 & Chordwise location of max. $t / c$ \\
\hline 10 & 2.838530 & 2.741494 & 1 & Airfoil LE radius parameter, $r_{t}$ \\
\hline 11 & 2.787242 & 2.710833 & 0.01 & Airfoil $t / c$ at root \\
\hline 12 & 2.102081 & 2.181458 & 0.01 & Airfoil $t / c$ at LE break \\
\hline 13 & 2.039613 & 2.153050 & 0.01 & Airfoil $t / c$ at tip \\
\hline 14 & 0.029378 & 0.029391 & 100 & Fuselage restraint $1, x(\mathrm{ft})$ \\
\hline 15 & 0.031241 & 0.029997 & 10 & Fuselage restraint $1, r(\mathrm{ft})$ \\
\hline 16 & 0.135445 & 0.135729 & 100 & Fuselage restraint $2, x(\mathrm{ft})$ \\
\hline 17 & 0.251432 & 0.250103 & 10 & Fuselage restraint $2, r(\mathrm{ft})$ \\
\hline 18 & 1.157109 & 1.142453 & 100 & Fuselage restraint $3, x(\mathrm{ft})$ \\
\hline 19 & 0.523216 & 0.531618 & 10 & Fuselage restraint $3, r(\mathrm{ft})$ \\
\hline 20 & 1.902365 & 1.873612 & 100 & Fuselage restraint $4, x(\mathrm{ft})$ \\
\hline 21 & 0.549794 & 0.546006 & 10 & Fuselage restraint $4, r(\mathrm{ft})$ \\
\hline 22 & 0.784794 & 0.777027 & 10 & Nacelle $1, x_{9}(\mathrm{ft})$ \\
\hline 23 & 3.510797 & 3.564725 & 10 & Nacelle $2, x_{10}(\mathrm{ft})$ \\
\hline 24 & 3.251852 & 3.311839 & 100000 & Mission fuel (lbs) \\
\hline 25 & 5.801989 & 5.772331 & 10000 & Starting cruise altitude (ft) \\
\hline 26 & 0.381966 & 0.377536 & 100 & Cruise climb rate $(\mathrm{ft} / \mathrm{min})$ \\
\hline 27 & 6.381778 & 6.779027 & 100 & Vertical tail area $\left(\mathrm{ft}^{2}\right)$ \\
\hline 28 & 6.841329 & 7.436056 & 100 & Horizontal tail area $\left(\mathrm{ft}^{2}\right)$ \\
\hline
\end{tabular}


Table E.4: Design variables for optimal HSCT design using RS3.

\begin{tabular}{|c|c|c|c|c|}
\hline$\#$ & $\overline{\mathrm{RS}} 3_{\mathrm{A}}$ & $\overline{\mathrm{RS}} 3_{\mathrm{AB}}$ & Multiplier & Description \\
\hline 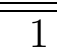 & 1.554487 & 1.561435 & 100 & Wing root chord (ft) \\
\hline 2 & 1.267094 & 1.236314 & 100 & LE Break, $x(\mathrm{ft})$ \\
\hline 3 & 3.864408 & 3.635826 & 10 & LE Break, $y(\mathrm{ft})$ \\
\hline 4 & 1.587124 & 1.563227 & 100 & TE Break, $x(\mathrm{ft})$ \\
\hline 5 & 5.234883 & 5.233100 & 10 & TE Break, $y(\mathrm{ft})$ \\
\hline 6 & 1.475031 & 1.442268 & 100 & LE of wing tip, $x(\mathrm{ft})$ \\
\hline 7 & 0.770903 & 0.759863 & 10 & Tip chord (ft) \\
\hline 8 & 6.030346 & 6.054834 & 10 & Wing semi-span (ft) \\
\hline 9 & 3.977452 & 4.055377 & 0.10 & Chordwise location of max. $t / c$ \\
\hline 10 & 3.279015 & 2.963885 & 1 & Airfoil LE radius parameter, $r_{t}$ \\
\hline 11 & 2.648976 & 2.736536 & 0.01 & Airfoil $t / c$ at root \\
\hline 12 & 1.787991 & 1.797197 & 0.01 & Airfoil $t / c$ at LE break \\
\hline 13 & 1.546993 & 1.606331 & 0.01 & Airfoil $t / c$ at tip \\
\hline 14 & 0.029135 & 0.029284 & 100 & Fuselage restraint $1, x(\mathrm{ft})$ \\
\hline 15 & 0.010777 & 0.011781 & 10 & Fuselage restraint $1, r(\mathrm{ft})$ \\
\hline 16 & 0.134883 & 0.135568 & 100 & Fuselage restraint $2, x(\mathrm{ft})$ \\
\hline 17 & 0.244995 & 0.245981 & 10 & Fuselage restraint $2, r(\mathrm{ft})$ \\
\hline 18 & 1.155367 & 1.155823 & 100 & Fuselage restraint $3, x(\mathrm{ft})$ \\
\hline 19 & 0.527971 & 0.527311 & 10 & Fuselage restraint $3, r(\mathrm{ft})$ \\
\hline 20 & 1.889478 & 1.891659 & 100 & Fuselage restraint $4, x(\mathrm{ft})$ \\
\hline 21 & 0.549109 & 0.549093 & 10 & Fuselage restraint $4, r(\mathrm{ft})$ \\
\hline 22 & 0.863997 & 0.823570 & 10 & Nacelle $1, x_{9}(\mathrm{ft})$ \\
\hline 23 & 3.868816 & 4.267067 & 10 & Nacelle $2, x_{10}(\mathrm{ft})$ \\
\hline 24 & 3.250141 & 3.294902 & 100000 & Mission fuel (lbs) \\
\hline 25 & 5.785371 & 5.752917 & 10000 & Starting cruise altitude (ft) \\
\hline 26 & 0.383243 & 0.387235 & 100 & Cruise climb rate $(\mathrm{ft} / \mathrm{min})$ \\
\hline 27 & 7.003306 & 7.545314 & 100 & Vertical tail area $\left(\mathrm{ft}^{2}\right)$ \\
\hline 28 & 6.874748 & 6.987987 & 100 & Horizontal tail area $\left(\mathrm{ft}^{2}\right)$ \\
\hline
\end{tabular}




\section{Appendix F}

\section{RSG Program}

The Response Surface Generation program RSG generates response surfaces using the method of least squares, Appendix A. RSG can be invoked by typing: rsa input.deck.

The file input.deck contains the basic control parameters for the operation of RSG. After the input deck has been read, RSG will load and pre-process any necessary data. Two sets of data are used within RSG, the fit data set and the compare data set. The former is used to generate the response surface while the latter is used to evaluate response surface accuracy. Messages are posted to the standard output describe RSG's progress. After solving the least squares problem, further information which describes the response surface can be printed to the screen and various output files.

\section{F.1 Data Input}

RSG reads information from three types of files, the input deck, the model file, the data file. The input deck contains the basic control parameters for RSG. The model file describes the response surface model function. Data files contain information about the data points used to generate and evaluate the response surface.

\section{The Input Deck}

RSG will not execute without a proper input deck. All data pre-processing instructions are included in this file as well as the locations of the model file and data files. The input deck file must appear in a specific format which is detailed later in 
this chapter.

\section{The Model File}

The model function is described within the model file and can appear in one of two formats, the JMP format and the standard format. The JMP format is identical to the model function output from JMP by SAS Institute Inc. 1 [34]; however, the variables must have the names $X 01, X 02, \ldots X N$. Refer to the JMP Manual for more details about this format.

The standard format [35] was developed to describe an arbitrary polynomial, where each terms appears in a linear fashion. It is based on the assumption that every term in the model function can be written as:

$$
c x_{1}^{a_{1}} x_{2}^{a_{2}} \cdots x_{p}^{a_{p}}
$$

where $x_{i}$ are the variables and $a_{i}$ are known constants.

The best way to describe the standard format it to begin with an example. Consider a quadratic polynomial in three variables given as:

$$
y=c_{1}+c_{2} x_{1}+c_{3} x_{2}+c_{4} x_{3}+c_{5} x_{1}^{2}+c_{6} x_{1} x_{2}+c_{7} x_{1} x_{3}+c_{8} x_{2}^{2} c_{9} x_{2} x_{3}+c_{10} x_{3}^{2} .
$$

The model file which describes this function appears in Figure F.1. The first line is a header which describes each column. This line and any other lines beginning with text in the standard model file are ignored by RSG. Column one gives the index number of each model function term and is also ignored by RSG. The following $p$ columns, where $p$ is the number of variables, represent the exponent of the individual variable within each model function term. If all variables are raised to the zero power, the model function term will be a constant. The final column is a text description of the term and is not required. The exponents do not have to be integers.

RSG can read and output either model file type. In addition, RSG can create standard model functions such as linear, quadratic and cubic polynomials. More details will be discussed in the input deck section.

\section{The Data File}

Data files contain information about the data points used to construct and evaluate the response surface. All required information can be stored in one data file, or it can 


\begin{tabular}{|ccccl|}
\hline Index & $x_{1}$ & $x_{2}$ & $x_{3}$ & Description \\
1 & 0 & 0 & 0 & constant \\
2 & 1 & 0 & 0 & $x_{1}$ \\
3 & 0 & 1 & 0 & $x_{2}$ \\
4 & 0 & 0 & 1 & $x_{3}$ \\
5 & 2 & 0 & 0 & $x_{1}^{2}$ \\
6 & 1 & 1 & 0 & $x_{1} x_{2}$ \\
7 & 1 & 0 & 1 & $x_{1} x_{3}$ \\
8 & 0 & 2 & 0 & $x_{2}^{2}$ \\
9 & 0 & 1 & 1 & $x_{2} x_{3}$ \\
10 & 0 & 0 & 2 & $x_{3}^{2}$ \\
\hline
\end{tabular}

Figure F.1: RSG standard model function file example.

be spread among several. For instance, the values of each variable might be stored in one file while the objective function values might be stored in another.

Each line of a data file must correspond to a separate data point. In addition, if more than one data file is used to describe a single point (eg. one data file contains values for the variables while another file contains the objective function values) then the lines of each data file must correspond to one another.

RSG allows comments to be placed throughout the data file as long as they do not appear on a line containing data. In addition, comments cannot begin with the characters $0 \ldots 9$ or the ' $\therefore$. Columns can be separated by tabs, spaces or commas. RSG can be instructed to use data from any column within the file; however, variables used for the response surface must be located in consecutive columns.

A typical data file appears in Figure F.2. The comment lines Experiment number 1 and Experiment number 2 are ignored by RSG. Column one is an index number for the data and is not used in the response surface generation. Columns two and three give values for the variables $x_{1}$ and $x_{2}$ associated with each data point. Objective function values $y$ for each data point are given in column four.

\section{F.2 Data Pre-Processing}

RSG performs several types of preprocessing which can be categorized by their effect on the data. Data clipping and replacing are designed to alter specific data points 


\begin{tabular}{|c|c|c|c|}
\hline Inde & & $x_{2}$ & $y$ \\
\hline $\operatorname{Exp}$ & nent & numl & er 1 \\
\hline 1 & 1.1 & 6.2 & 2.1 \\
\hline 2 & 3.2 & 6.0 & 3.6 \\
\hline 3 & 2.1 & 4.8 & 1.7 \\
\hline $\operatorname{Exp}$ & nent & numb & er 2 \\
\hline 4 & 8.5 & 0.4 & 0.1 \\
\hline 5 & 9.0 & 1.3 & 0.4 \\
\hline 6 & 7.8 & 1.7 & 0.2 \\
\hline
\end{tabular}

Figure F.2: RSG data file example.

while leaving the rest unchanged. Together, these operations can be considered point procedures. Global procedures are those which change the entire data set and include scaling and conversion to a $\log$ scale. Note that after executing the least squares method, RSG reverses the effect of the global procedures. Therefore, RSG's output is always in the same scale as the input. This section describes each procedure in the order which they are performed by RSG.

\section{Clip Data}

Sometimes it is advantageous to remove specific data points from those used to create or evaluate a response surface. Two methods are provided for removing points. First, points can be removed based on their position in a data file. For example, you could remove all of the points appearing before the 100th and after the 175th entry in the data file.

A more useful way to remove points is to pick a column of data, the clip reference column and then evaluate each entry in that column. If the entry falls outside of a specified range, then the data point associated with that column will be removed. RSG allows you to pick an arbitrary column of data (in any file) and remove data points when the specified column value falls above or below a specified limit.

\section{Replace Data}

An alternative to clipping data points is to replace the objective function value with one which is preselected. RSG allows you to specify a range of acceptable values for a column of data, the replace reference column. If the column's entry falls outside of that range, the objective function is replaced. The value used to replace the 
objective function depends on whether the replace reference column entry is above or below the acceptable range.

\section{Switch to Log Scale}

RSG can automatically convert data to the natural log scale. This can be done for either the objective function, the variables, or both. Data which assumes values below zero cannot be converted; therefore, another pre-processing procedure is available which allows you to shift all of the data above a minimum value.

\section{Scale Data}

To prevent ill conditioning due to variables which have varying orders of magnitude, RSG can be instructed to scale data between -1 and 1 . To properly scale the design variables and/or the objective function, RSG must know the extremum values for the variables and objective function. The values can be based on the fit data, the compare data, or both. If a single response surface will be evaluated with multiple compare data sets, only the fit data should be used to find the extremum values. This guarantees that the response surface will be identical for regardless of the compare data.

\section{F.3 Response Surface Generation}

Once the variables and objective function have been pre-processed, the response surface can be generated. RSG offers four methods to create the response surface; however, each will produce identical results for well conditioned systems. In addition, RSG can solve the weighted least squares problem. Once the response surface has been created, RSG can remove unimportant terms using regression analysis and analysis of variance (ANOVA).

\section{Weighted Least Squares}

Weightings can be added to each data point before the response surface is produced in one of two ways. First, RSG can be instructed to use a column of data as the weighting factor. Second, RSG can be instructed to create a normal distribution curve with a given mean and standard deviation. Weighting factors are then taken 
from this curve at an ordinate position corresponding to predefined column of data.

\section{Least Squares Algorithms}

RSG implements two least squares algorithms (Appendix A) from the LAPACK library [36]. One uses singular value decomposition (SVD) and the other uses QR Factorization (QR). Although slower, SVD is more robust and is the preferred method. For each algorithm, RSG can either work with the regular equations or the normal equations (NE). RSG can automatically choose between the two SVD algorithms depending on the amount of memory required.

\section{Regression Analysis and ANOVA}

Regression analysis and ANOVA are statistical techniques which enable the less significant terms in the model function to be identified. In this process, the term with the highest coefficient of variation (see Appendix A) is removed from the model function, and the process of regression analysis and ANOVA is repeated until a stopping criteria reached. RSG will discontinue this process when a specified number of terms in the model function remain. Removing one term at a time is known as one-step backwards regression [6].

\section{F.4 Data Output}

After generating the response surface, RSG produces various types of output information. Most output can be turned on or off from the input deck. When appropriate, RSG reverses the global pre-processing procedures.

\section{Response Surface Accuracy}

At the completion of the least squares algorithm, RSG determines the response surface accuracy by evaluating it at a series of data points, the compare data set. The compare data may be different from the data used to generate the response surface. Results are given in the same scale as the original objective functions. Information produced includes the average error, the RMS error, and the maximum Error.

If the SVD method is used to generate the response surface, the determinant of $\mathbf{X}^{T} \mathbf{X}$ and the condition number of $\mathbf{X}$ are also reported. The determinant can be used 
as a measure of the quality of points used for generating the curve fit; however, this value is highly dependent on the number of data points used. Therefore, a scaled version of the determinant is printed [6]:

$$
\frac{\left|\mathbf{X}^{T} \mathbf{X}\right|}{n^{p}} .
$$

where $n$ is the number of data points used to create the response surface and $p$ is the number of variables in the model function. For large problems, the scaled determinant will approach zero. Therefore, RSG reports the scaled determinant raised to the 1/4 power.

\section{Response Surface Description}

A description of the response surface can be printed in the standard model file format or the JMP model file format. Information about each term in the model function can also be printed. This includes the coefficient value, the standard deviation and the coefficient of variation associated with each term.

\section{Response Surface Code}

RSG can generate source code for $\mathrm{C} / \mathrm{C}++$, Fortran 77, and Mathematica. These codes take into account global pre-processing.

\section{Other Types of Output}

The error associated with each point in the compare data set can be printed to a file. The exact form of the data which enters the least squares algorithm can be printed to a file. And, input for GRAMPS [35], a program for evaluating D-Optimality, can also be produced by RSG.

\section{F.5 RSG Input Deck}

The input deck specifies how RSG should operate and where other input files are located. Each section of the input deck file must appear in a specific format; however, the sections of the input deck may appear in any order. RSG identifies each section by searching for the section header, words in all capitals which begin with an underscore (_). After the line which delineates the beginning of the section, RSG reads the first 
column of data and ignores the remainder of the line. The following is a description of each section and a description of each input parameter. Note that not all of the sections are required in the input deck. RSG will alert you if any information is missing.

\section{General Instructions}

\section{-GENERAL}

par_1 Number of variables

par_2 Fit \& compare data are identical (0: False, 1: True)

par_3 Fit \& compare clip/replace are identical (0: False, 1: True)

par_4 Base global pre-process on which data set (0: Compare, 1: Fit, 2: Both)

par_5 Least squares algorithm (0: Auto, 1: QR, 2: SVD, 3: QR NE, 4: SVD NE)

RSG supports two least squares algorithms, singular value decomposition (SVD) and QR Factorization (QR). For each method, RSG can work with either the regular equations or the normal equations (NE). If given the opportunity, RSG will automatically choose between SVD and SVD NE based on the amount of memory required.

\section{Location of Fit Data}

\begin{tabular}{|cl|}
\hline FIT_DATA & \\
par_1 & Name of file with the variables \\
par_2 & Name of file with objective function values \\
par_3 & Name of file with clip reference column \\
par_4 & Name of file with replace reference column \\
par_5 & Name of file with weighted least squares reference column \\
par_6 & Column number with the first variable \\
par_7 & Column number with the objective function \\
par_8 & Column number with the clip reference \\
par_9 & Column number with the replace reference \\
par_10 & Column number with the weighted least squares reference \\
\hline
\end{tabular}

All files specified in this section must adhere to the data file format. Files will only be loaded if necessary and they will only be loaded once even if the same file name is entered multiple times. 
If RSG has been directed to use the same data for both the fit and compare data set, information from this section will be used to both create and evaluate the response surface. A similar section, labeled _COMPARE_DATA, must be included if the compare data is different.

\section{Clipping Instructions}

$$
\begin{array}{ll}
\text { _CLIP_FIT_DATA } \\
\text { par_1 } & \text { Clip data (0: False, 1: True) } \\
\text { par_2 } & \text { Clip if reference value is below this value } \\
\text { par_3 } & \text { Clip if reference value is above this value } \\
\text { par_4 } & \text { Clip if position in file is below this value } \\
\text { par_5 } & \text { Clip if position in file is above this value }
\end{array}
$$

If RSG has been directed to use the same clip/replace options for both the fit and compare data set, information from this section will be used for both fit and compare sets. A similar section, labeled _CLIP_COMPARE_DATA, must be included if the compare data is to be treated differently.

\section{Replace Data Instructions}

_REPLACE_FIT_OBJECTIVE

par_1 Replace data (0: False, 1: True)

par 2 Replace objective function if reference value is below this value

par_3 Replace objective function if reference value is above this value

par_4 If the reference value is low set the objective function to this value

par_5 If the reference value is high set the objective function to this value

If RSG has been directed to use the same clip/replace options for both the fit and compare data set, information from this section will be used for both fit and compare sets. A similar section, labeled_REPLACE_COMPARE_OBJECTIVE, must be included if the compare data is to be treated differently. 


\section{Pre-process Variables Instructions}

\section{PREPROCESS_VARIABLES}

par_1 Shift each variable above minimum value (0: False, 1: True)

par_2 Minimum value for shift

par_3 Take $\ln ()$ of variables (0: False, 1: True)

par_4 Scale variables to values $-1 \cdots 1$ (0: False, 1 : True)

\section{Pre-process Objective Function Instructions}

\section{_PREPROCESS_OBJECTIVE}

par_1 Shift objective above minimum value (0: False, 1: True)

par_2 Minimum value for shift

par_3 Take $\ln ()$ of objective (0: False, 1: True)

par_4 Scale objective to values $-1 \cdots 1$ (0: False, 1: True)

\section{Weighted Least Squares Instructions}

\section{_WEIGHTED_LEAST_SQUARES}

par_1 Apply weighting to fit data (0: False, 1: True)

par_2 Source of weighting (1: Data file, 2: Normal distribution)

par_3 Normal distribution mean value

par_4 Normal distribution standard deviation

par_5 Use weighting in error calculation (0: False, 1: True)

\section{Backward Regression Instructions}

BACKWARD_REGRESSION Information

par_1 Perform backwards regression (0: False, 1: True)

par_2 Minimum number of terms allowed in model function

par_3 Number of coefficients to remove during each iteration

par_4 Allow removal of linear terms (0: False, 1: True) 


\section{Screen Output Instructions}

\begin{tabular}{|cl|}
\hline SCREEN_OUTPUT \\
par_1 & Print standard RS description (0: False, 1: True) \\
par_2 & Print RS properties (0: False, 1: True) \\
par_3 & Print C/C++ source code (0: False, 1: True) \\
par_4 & Print f77 source code (0: False, 1: True) \\
par_5 & Print Mathematica source code (0: False, 1: True) \\
par_6 & Print JMP RS description (0: False, 1: TRUE) \\
par_6 & Print LaTeX RS description (0: False, 1: TRUE) \\
\hline
\end{tabular}

\section{File Output Instructions}

\section{_FILE_OUTPUT}

par_1 Output a GRAMPS input file (0: False, 1: True)

par_2 Include global pre-processing in GRAMPS input file (0: False, 1: True)

par_3 Output the error at each compare point (0: False, 1: True)

par_4 Include clipped data in error output (0: False, 1: True)

par_5 Output the pre-processed data (0: False, 1: True)

par_6 Root file name for output files

\section{Model Function Description}

\section{_MODEL_DESCRIPTION}

par_1 Source (0: Input Deck, 1: Standard model file, 2: JMP model file)

par_2 Name of model function input file

\section{Input Deck Model Function Description}

\section{INPUT_DECK_MODEL}

par_1 Model type (1: Linear, 2: Quadratic, 3: Cubic, 99: User defined)

This section is only required if the source of the model function is set to the input deck in the _MODEL_DESCRIPTION section. Response surface models can be created automatically by RSG or a standard model function description can appear immediately following this section. 


\section{Appendix G}

\section{Vitae}

The author was born in Los Angeles on March 7th, 1971. After graduating from Saratoga High School in 1989, he attended the University of California at Davis. Graduating with high honors in June of 1994, the author received bachelor degrees in both aeronautical and mechanical engineering. Immediately following the completion of his undergraduate work, the author pursued graduate studies at Virginia Polytechnic Institute and State University. After completing a masters of science degree in aerospace engineering in March of 1996, the author began a career with the Missiles and Space Division of Lockheed/Martin. 